\title{
COMBINATORIAL DICHOTOMIES AND CARDINAL INVARIANTS
}

\author{
Dilip Raghavan and Stevo Todorcevic
}

\begin{abstract}
Assuming the P-ideal dichotomy, we attempt to isolate those cardinal characteristics of the continuum that are correlated with two well-known consequences of the proper forcing axiom. We find a cardinal invariant $\mathfrak{x}$ such that the statement that $\mathfrak{x}>\omega_{1}$ is equivalent to the statement that $1, \omega, \omega_{1}, \omega \times \omega_{1}$, and $\left[\omega_{1}\right]^{<\omega}$ are the only cofinal types of directed sets of size at most $\aleph_{1}$. We investigate the corresponding problem for the partition relation $\omega_{1} \rightarrow\left(\omega_{1}, \alpha\right)^{2}$ for all $\alpha<\omega_{1}$. To this effect, we investigate partition relations for pairs of comparable elements of a coherent Suslin tree $\mathbb{S}$. We show that a positive partition relation for such pairs follows from the maximal amount of the proper forcing axiom compatible with the existence of $\mathbb{S}$. As a consequence, we conclude that after forcing with the coherent Suslin tree $\mathbb{S}$ over a ground model satisfying this relativization of the proper forcing axiom, $\omega_{1} \rightarrow\left(\omega_{1}, \alpha\right)^{2}$ for all $\alpha<\omega_{1}$. We prove that this positive partition relation for $\mathbb{S}$ cannot be improved by showing in ZFC that $\mathbb{S} \nrightarrow\left(\aleph_{1}, \omega+2\right)^{2}$.
\end{abstract}

\section{Introduction}

An interesting, though lesser known, phenomenon in set theory is that cardinal invariants of the continuum can be used to calibrate the strength of various mathematical propositions (that do not unnecessarily involve sets of reals) in the presence of certain kinds of combinatorial dichotomies. These mathematical statements are invariably consequences of forcing axioms such as Proper Forcing Axiom (PFA) or Martin's Maximum (MM), and they are negated by Continuum Hypothesis $(\mathrm{CH})$. The combinatorial dichotomies we are interested in are compatible with $\mathrm{CH}$, but they do keep a considerable amount of the strength of PFA or MM by, for example, negating square-principles or reflecting stationary sets. In fact, they have a tendency of pushing several mathematical statements down to concrete questions about combinatorial properties of sets of reals that seem to be expressible in terms of cardinal invariants of the continuum. A typical theorem of the sort we have in mind looks as follows.

Prototypical theorem. Assume CD. Then the following are equivalent:

(1) $\mathfrak{x}>\omega_{1}$.

(2) $\phi$.

Here $\phi$ is some mathematical statement, $\mathfrak{x}$ is a cardinal invariant, and CD is a combinatorial dichotomy that is consistent with $\mathrm{CH}$. As relations between cardinal invariants have been well-investigated, theorems like this permit us to calibrate the

Received by the editors November 1, 2013.

2010 Mathematics Subject Classification. 03E05, 03E17, 03E65.

Key words and phrases. combinatorial dichotomies, partition relation, $\mathrm{P}$-ideal dichotomy, cardinal invariants, coherent Suslin tree, Laver property. 
relative strength of various mathematical propositions over the base theory ZFC $+\mathrm{CD}$ (ZFC is Zermelo-Fraenkel set theory with the axiom of choice). Examples of CD include Rado's Conjecture (RC) and the P-ideal dichotomy (PID). The statement $\phi$ can come from different areas of mathematics. We illustrate this with two recent typical examples.

Theorem 1 (Todorčević and Torres Pérez [23]). Assume RC. Then the following are equivalent:

(1) $\mathfrak{c}>\omega_{1}$.

(2) There are no special $\omega_{2}$-Aronszajn trees.

Here $\mathfrak{c}$ is the size of the continuum, the most basic of cardinal invariants.

Theorem 2 (Brech and Todorčević [3]). Assume PID. Then the following are equivalent:

(1) $\mathfrak{b}>\omega_{1}$.

(2) Every non-separable Asplund space has an uncountable almost bi-orthogonal system.

Here $\mathfrak{b}$ is the bounding number; its precise definition is given in Section 2. For more regarding the big picture surrounding such results, consult [21].

The purpose of this paper is to add to the analysis of PID from this point of view; we do not have results about RC here. PID is a well-known consequence of PFA that is consistent with CH. (See Section 2 below where we give a definition of PID.) Indeed, it is strong enough to imply many of the consequences of PFA that do not contradict $\mathrm{CH}$. For example, PID implies that there are no Suslin trees (see [1]), it implies that $\square(\theta)$ fails for every ordinal $\theta$ of cofinality $>\omega_{1}$ (see [19]), it implies the singular cardinals hypothesis (see [25]), and it implies that $\square_{\kappa, \omega}$ fails for all uncountable cardinals $\kappa$ (see [12]). For many consequences of PFA contradicting $\mathrm{CH}$, PID tends to reduce the amount of PFA involved to the hypothesis that some cardinal invariant of the continuum is bigger than $\omega_{1}$. The pseudo-intersection number $\mathfrak{p}$ (see Section 2 for definition), being smaller than most of the usual cardinals, almost always suffices. We are interested in finding out the precise cardinal invariant which is needed for several specific consequences of PFA. So our general project is twofold:

General Problem 1. Given a statement $\phi$ which is a consequence of PID $+\mathrm{MA}_{\aleph_{1}}$, find a cardinal invariant $\mathfrak{x}$ such that $\phi$ is equivalent to $\mathfrak{x}>\omega_{1}$ over $\mathrm{ZFC}+$ PID.

For example, if $\phi$ is the statement that every non-separable Asplund space has an uncountable almost bi-orthogonal system (see [6] for definitions), then it is a theorem of Todorčević [20] that PID $+\mathrm{MA}_{\aleph_{1}}$ implies $\phi$, while another result of Todorčević (see [18], Chapter 2) shows that $\phi$ implies $\mathfrak{b}>\omega_{1}$. So the result of Theorem 2 above came as an answer to this version of the general problem.

General Problem 1 asks if the influence of PFA on $\phi$ can be decomposed into a part which is consistent with $\mathrm{CH}$ and into another $\mathrm{CH}$ violating part that is precisely captured by the cardinal invariant $\mathfrak{x}$. This reveals the nature of the combinatorial phenomenon on the reals needed for $\phi$. Another motivation is that one often has to find a new and sharper proof of $\phi$ in order to accomplish this project. A slightly less ambitious project is 
General Problem 2. Given a statement $\phi$ which is a consequence of $\mathrm{PID}+\mathfrak{p}>\omega_{1}$, investigate whether $\phi$ is equivalent to $\mathfrak{p}>\omega_{1}$ over $\mathrm{ZFC}+\mathrm{PID}$.

A canonical model for investigating this can be obtained by forcing with a coherent Suslin tree $\mathbb{S}$ over a model of PFA(S) (see Section 2 for the precise definition of a coherent Suslin tree). Here PFA(S) is the maximal amount of PFA that is consistent with the existence of $\mathbb{S}$.

Definition 3. Let $\mathbb{S}$ be a coherent Suslin tree. PFA $(\mathbb{S})$ is the following statement. If $\mathbb{P}$ is a poset which is proper and preserves $\mathbb{S}$ and $\left\{D_{\alpha}: \alpha<\omega_{1}\right\}$ is a collection of dense subsets of $\mathbb{P}$, then there is a filter $G$ on $\mathbb{P}$ such that $\forall \alpha<\omega_{1}\left[G \cap D_{\alpha} \neq 0\right]$.

The consistency of PFA $(\mathbb{S})$ can be proved assuming the existence of a supercompact cardinal by iterating with countable support all proper posets which preserve $\mathbb{S}$. One obtains an interesting model where a large number of consequences of PFA hold by forcing with $\mathbb{S}$ over a ground model satisfying PFA(S). In particular, PID holds in this extension. Moreover, almost all of the cardinal invariants of the continuum are equal to $\omega_{2}$. However, $\mathfrak{p}=\omega_{1}$. Therefore, if a consequence of PFA is consistent with PID $+\mathfrak{p}=\omega_{1}$, then it is very likely to be true in this model. Thus this model is useful for providing negative answers to the General Problem 2 above.

In this paper, we investigate two well-known consequences of PFA in view of these two general problems. The first one concerns Tukey theory. Recall that a poset $\langle D, \leq\rangle$ is directed if any two members of $D$ have an upper bound in $D$. A set $X \subset D$ is unbounded in $D$ if it does not have an upper bound in $D$. A set $X \subset D$ is said to be cofinal in $D$ if $\forall y \in D \exists x \in X[y \leq x]$. Given directed sets $D$ and $E$, a map $f: D \rightarrow E$ is called a Tukey map if the image of every unbounded subset of $D$ is unbounded in $E$. A map $g: E \rightarrow D$ is called a convergent map if the image of every cofinal subset of $E$ is cofinal in $D$. It is easy to see that there is a Tukey map $f: D \rightarrow E$ iff there exists a convergent $g: E \rightarrow D$. When this situation obtains, we say that $D$ is Tukey reducible to $E$, and we write $D \leq_{T} E$. This induces an equivalence relation on directed posets in the usual way: $D \equiv_{T} E$ iff both $D \leq_{T} E$ and $E \leq_{T} D$. If $D \equiv_{T} E$, we say that $D$ and $E$ are Tukey equivalent or have the same cofinal type, and this is intended to capture the idea that $D$ and $E$ have "the same cofinal structure." As support for this, it can be shown that $D \equiv_{T} E$ iff there is a directed set $R$ into which both $D$ and $E$ embed as cofinal subsets, so that $D$ and $E$ describe the same cofinal type, the one of $R$.

These notions first arose in the Moore-Smith theory of convergence studied by general topologists (see $[8,24]$ ). The following result of Todorcevic gives a classification of the possible cofinal types of directed posets of size at most $\aleph_{1}$ under PFA.

Theorem 4 (Todorčević [16]). Under PID $+\mathfrak{p}>\omega_{1}$, there are only five Tukey types of size at most $\aleph_{1}: 1, \omega, \omega_{1}, \omega \times \omega_{1}$, and $\left[\omega_{1}\right]^{<\omega}$.

Here, the ordering on $\omega \times \omega_{1}$ is the product ordering and $\left[\omega_{1}\right]^{<\omega}$ is ordered by inclusion. In Section 4, we solve General Problem 1 for the statement that 1, $\omega$, $\omega_{1}, \omega \times \omega_{1}$, and $\left[\omega_{1}\right]^{<\omega}$ are the only cofinal types of size at most $\aleph_{1}$. Interestingly, the cardinal invariant that captures this statement turns out not to be one of the commonly occurring ones; rather, it is the minimum of two mutually independent cardinals. The result in this section answers both Questions 24.14 and 24.17 of [21], 
which ask whether the statement that $1, \omega, \omega_{1}, \omega \times \omega_{1}$, and $\left[\omega_{1}\right]^{<\omega}$ are the only cofinal types of size at most $\aleph_{1}$ is equivalent over ZFC + PID to $\mathfrak{b}=\omega_{2}$ and to $\mathfrak{p}=\omega_{2}$, respectively. The answer to both questions turns out to be "no."

In Section 3, we investigate a strong version of the ordinary partition relation on $\omega_{1}$, namely the relation $\omega_{1} \rightarrow\left(\omega_{1}, \alpha\right)^{2}$. Recall that for an ordinal $\alpha, \omega_{1} \rightarrow\left(\omega_{1}, \alpha\right)^{2}$ means that for any $c:\left[\omega_{1}\right]^{2} \rightarrow 2$ either there exists $X \in\left[\omega_{1}\right]^{\omega_{1}}$ such that $c^{\prime \prime}[X]^{2}=\{0\}$ or there exists $X \subset \omega_{1}$ such that $\operatorname{otp}(X)=\alpha$ and $c^{\prime \prime}[X]^{2}=\{1\}$. The DushnikMiller theorem says that $\omega_{1} \rightarrow\left(\omega_{1}, \omega\right)^{2}$ (see [4]), and its strengthening proved by Erdős and Rado states that $\omega_{1} \rightarrow\left(\omega_{1}, \omega+1\right)^{2}$ (see [5]), while the classical coloring of Sierpinski [13] shows that $\omega_{1} \rightarrow\left(\omega_{1}, \omega_{1}\right)^{2}$ is false. Thus the following theorem of Todorčević [15] gives the strongest possible version of the ordinary partition relation on $\omega_{1}$.

Theorem 5 (Todorcevic). PID $+\mathfrak{p}>\omega_{1}$ implies that $\omega_{1} \rightarrow\left(\omega_{1}, \alpha\right)^{2}$, for every $\alpha<\omega_{1}$.

This should be compared with the following result of Todorčević [18] (Chapter 2) that is relevant to General Problem 1.

Theorem 6 (Todorcevic). $\mathfrak{b}=\omega_{1}$ implies $\omega_{1} \nrightarrow\left(\omega_{1}, \omega+2\right)^{2}$.

In Section 3, we solve General Problem 2 for the statement that $\omega_{1} \rightarrow\left(\omega_{1}, \alpha\right)^{2}$, for every $\alpha<\omega_{1}$ by showing that it holds after forcing with the coherent Suslin tree $\mathbb{S}$ over a ground model satisfying $\mathrm{PFA}(\mathbb{S})$. This shows that $\mathfrak{p}>\omega_{1}$ is not equivalent over $\mathrm{ZFC}+\mathrm{PID}$ to the statement that $\omega_{1} \rightarrow\left(\omega_{1}, \alpha\right)^{2}$ for every $\alpha<\omega_{1}$ so it remains to look for another cardinal invariant of the continuum that would capture this partition relation for $\omega_{1}$.

\section{Notation}

We set up some basic notation that will be used throughout the paper. " $a \subset b$ " means $\forall x[x \in a \Longrightarrow x \in b]$, so the symbol " $\subset$ " does not denote proper subset. " $\forall \infty$ " means for all but finitely many and " $\exists \infty$ " stands for there exists infinitely many.

Definition 7. Let $X$ be an uncountable set. An ideal $\mathcal{I} \subset[X]^{\leq \omega}$ is called a $P$ ideal if for every countable collection $\left\{x_{n}: n \in \omega\right\} \subset \mathcal{I}$, there is $x \in \mathcal{I}$ such that $\forall n \in \omega\left[x_{n} \subset^{*} x\right]$.

All ideals are assumed to be non-principal, meaning that $[X]^{<\omega} \subset \mathcal{I}$.

Definition 8. The PID is the following statement: for any P-ideal $\mathcal{I}$ on an uncountable set $X$ either

(1) there is an uncountable set $Y \subset X$ such that $[Y]^{\leq \omega} \subset \mathcal{I}$

or

(2) there exist $\left\{X_{n}: n \in \omega\right\}$ such that the $X_{n}$ are pairwise disjoint, $X=\bigcup_{n \in \omega} X_{n}$, and $\forall n \in \omega\left[\left[X_{n}\right]^{\omega} \cap \mathcal{I}=0\right]$.

The following well-known cardinal invariants will occur throughout the paper. For functions $f, g \in \omega^{\omega}, f<^{*} g$ means $\forall^{\infty} n \in \omega[f(n)<g(n)]$. A set $F \subset \omega^{\omega}$ is said to be unbounded if there is no $g \in \omega^{\omega}$ such that $\forall f \in F\left[f<^{*} g\right]$. For sets $a$ and $b, a \subset^{*} b$ iff 
$a \backslash b$ is finite. A family $F \subset[\omega]^{\omega}$ is said to have the finite intersection property (FIP) if for any $A \in[F]^{<\omega}, \bigcap A$ is infinite.

Definition 9. c denotes $2^{\omega}$. Additionally,

$$
\begin{aligned}
& \mathfrak{p}=\min \left\{|F|: F \subset[\omega]^{\omega} \wedge F \text { has the FIP } \wedge \neg \exists b \in[\omega]^{\omega} \forall a \in F\left[b \subset^{*} a\right]\right\} . \\
& \mathfrak{b}=\min \left\{|F|: F \subset \omega^{\omega} \wedge F \text { is unbounded }\right\} .
\end{aligned}
$$

$\operatorname{cov}(\mathcal{M})$ is the least $\kappa$ such that $\mathbb{R}$ can be covered by $\kappa$ many meager sets.

It is easy to show $\omega_{1} \leq \mathfrak{p} \leq \mathfrak{b} \leq \mathfrak{c}$. Moreover, $\mathfrak{p} \leq \operatorname{cov}(\mathcal{M}) \leq \mathfrak{c}$, while $\mathfrak{b}$ and $\operatorname{cov}(\mathcal{M})$ are independent.

We will frequently make use of elementary submodels. We will simply write " $M \prec$ $H(\theta)$ " to mean " $M$ is an elementary submodel of $H(\theta)$, where $\theta$ is a regular cardinal that is large enough for the argument at hand."

Recall that a Suslin tree is an uncountable tree with no uncountable chains or antichains. We will use the following notation when dealing with a tree $\mathbb{S}$. For $t \in \mathbb{S}$, ht $(t)$ is the height of $t$. For an ordinal $\alpha$, let $\mathbb{S}_{\alpha}=\{s \in \mathbb{S}: \operatorname{ht}(s)=\alpha\}$ and $\mathbb{S}_{<\alpha}=$ $\{s \in \mathbb{S}: \operatorname{ht}(s)<\alpha\}$. For $t \in \mathbb{S}, \operatorname{pred}(t)$ denotes the set of predecessors of $t$, that is $\{s \in \mathbb{S}: s \leq t\}$. For a set $X$ and $t \in \mathbb{S}$, $\operatorname{pred}_{X}(t)=\operatorname{pred}(t) \cap X$. Dually, cone $(t)$ denotes the cone above $t$, for all $t \in \mathbb{S}$. In other words, cone $(t)=\{u \in \mathbb{S}: t \leq u\}$. Similarly, for a set $X$ and $t \in \mathbb{S}$, cone $_{X}(t)=\operatorname{cone}(t) \cap X$. Next, for $t \in \mathbb{S}$, $\operatorname{succ}(t)=\{u \in \mathbb{S}: u>$ $t$ and $h t(u)=h t(t)+1\}$. Once again, for $t \in \mathbb{S}$ and a set $X, \operatorname{succ}_{X}(t)=\operatorname{succ}(t) \cap X$. If $\mathbb{S}$ happens to be a normal tree, then for any non-empty $F \subset \mathbb{S}, \wedge F$ denotes the greatest lower bound in $\mathbb{S}$ of the elements of $F$.

Throughout the paper, we work with a fixed Suslin tree $\mathbb{S}$. We assume that $\mathbb{S}$ is a coherent strongly homogeneous Suslin tree. More precisely, this means that

(1) $\mathbb{S}$ is a Suslin tree and is a subtree of $\omega^{<\omega_{1}}$.

(2) For each $s \in \mathbb{S}, \exists^{\infty} n \in \omega[s \frown\langle n\rangle \in \mathbb{S}]$ and $\{t \in \mathbb{S}: t \geq s\}$ is uncountable.

(3) $\forall s, t, \in \mathbb{S}[|\xi \in \operatorname{dom}(s) \cap \operatorname{dom}(t): s(\xi) \neq t(\xi)|<\omega]$ (coherence).

(4) For each $\xi<\omega_{1}$ and $s, t \in \mathbb{S}_{\xi}$, there is an automorphism $\phi: \mathbb{S} \rightarrow \mathbb{S}$ such that $\phi(s)=t$ and $\forall \alpha \geq \xi \forall u \in \mathbb{S}_{\alpha}[\phi(u)=\phi(u \uparrow \xi) \cup u \uparrow[\xi, \alpha)]$ (strong homogeneity).

Thus fix once and for all a coherent strongly homogeneous Suslin tree $\mathbb{S}$. Though we remark that the coherence and strong homogeneity of $\mathbb{S}$ will not be needed until the proof of Lemma 19.

We will be studying colorings of the pairs of comparable elements of $\mathbb{S}$. We set up some basic notation relevant to such colorings here. For any $A, B \subset \mathbb{S}, A \otimes B=$ $\{\{a, b\}: a \in A$ and $b \in B$ and $a<b\} . A^{[2]}=A \otimes A$. The following variation of $A^{[2]}$ will also be important in Section 3. Let $Y \subset \mathbb{S}$ and $g: Y \rightarrow \mathbb{S}$. Then $Y_{g}^{[2]}$ denotes $\{\{a, b\}: a, b \in Y$ and $a<b$ and $g(a) \leq b\}$. If $S \subset \mathbb{S}$ and $c: S^{[2]} \rightarrow 2$ is a coloring, then $K_{i, c}=\left\{\{s, t\} \in S^{[2]}: c(\{s, t\})=i\right\}$, for each $i \in 2$. We will often omit the subscript " $c$ " when it is clear from the context.

We will use a $C$-sequence in the proof of Theorem 14. Recall that $\left\langle c_{\alpha}: \alpha<\omega_{1}\right\rangle$ is called a $C$-sequence if for each $\alpha<\omega_{1}$,

(1) $c_{\alpha} \subset \alpha$.

(2) If $\alpha$ is a limit ordinal, then $\operatorname{otp}\left(c_{\alpha}\right)=\omega$ and $\sup \left(c_{\alpha}\right)=\alpha$.

(3) If $\beta=\alpha+1$, then $c_{\beta}=\{\alpha\}$. 
Given a $C$-sequence $\left\langle c_{\alpha}: \alpha<\omega_{1}\right\rangle$, it is sometimes useful to think of each $c_{\alpha}$ as a function. For a fixed $0<\beta<\omega_{1}$ and $n \in \omega$, we adopt the following conventions. If $\beta=\alpha+1$, then $c_{\beta}(n)$ is the unique element of $c_{\beta}$, namely $\alpha$. If $\beta$ is a limit ordinal, then $c_{\beta}(n)$ is the $n$th element of $c_{\beta}$.

In the proof of Theorem 28, we will use the following notation for subtrees of $\omega^{<\omega}$. Given $T \subset \omega^{<\omega}$ which is a subtree, $[T]=\left\{f \in \omega^{\omega}: \forall n \in \omega[f\lceil n \in T]\}\right.$. For $\sigma \in T$, $\operatorname{succ}_{T}(\sigma)$ denotes $\{n \in \omega: \sigma \frown\langle n\rangle \in T\}$. Note that this departs from the definition of $\operatorname{succ}(s)$ when $s$ is a member of $\mathbb{S}$. However, since it will be clear when we are talking about members of $\mathbb{S}$ and when we are referring to elements of some subtree $T$ of $\omega^{<\omega}$, we hope that this will not cause any confusion.

\section{Partition relations after forcing with a coherent Suslin tree}

In this section, we investigate partition relations for the pairs of comparable elements of $\mathbb{S}$. Partition relations for the pairs of comparable elements of a Suslin tree were studied by Máté [11], and for more general partial orders by Todorčević [17]. We prove a positive partition relation for $\mathbb{S}^{[2]}$ assuming $\operatorname{PFA}(\mathbb{S})$ (Theorem 14). This result in an analog of Todorčević's theorem from [15] that PFA implies that $\omega_{1} \rightarrow\left(\omega_{1}, \alpha\right)^{2}$, for every $\alpha<\omega_{1}$. However, it is not a perfect analog. This is explained by Theorem 28, which establishes a negative partition relation for $\mathbb{S}^{[2]}$ in ZFC. This negative partition relation may be seen as a ZFC analog of the relation $\omega_{1} \not \nrightarrow\left(\omega_{1}, \omega+2\right)^{2}$ for the pairs of comparable elements of $\mathbb{S}$. It is somewhat surprising that such a result can be proved not going beyond ZFC. A corollary of the positive partition relation proved in this section is that $\omega_{1} \rightarrow\left(\omega_{1}, \alpha\right)^{2}$ for every $\alpha<\omega_{1}$ after forcing with $\mathbb{S}$ over a model of $\operatorname{PFA}(\mathbb{S})$.

In this section, all the trees we deal with will be subsets (though not subtrees) of $\mathbb{S}$. Of course, given $T \in[\mathbb{S}]^{\omega_{1}},\langle T, \leq\rangle$ is an $\omega_{1}$ tree with no uncountable chains or antichains. However to avoid some trivialities, we make the following definition:

Definition 10. $T \subset \mathbb{S}$ is called a Suslin tree if $T$ is uncountable and

(1) $\exists \min (T) \in T \forall x \in T[\min (T) \leq x]$.

(2) $\forall x \in T[\{y \in T: y \geq x\}$ is uncountable $]$.

Note that we are not requiring $T$ to be a normal tree. In general, $T$ will not be a subtree of $\mathbb{S}$.

Obviously, $\forall Y \in\left[\mathbb{S}^{\omega_{1}} \exists T \in[Y]^{\omega_{1}}[T\right.$ is a Suslin tree]. As this fact will be used frequently, we state it as a lemma.

Lemma 11. $\forall Y \in[\mathbb{S}]^{\omega_{1}} \exists T \in[Y]^{\omega_{1}}[T$ is a Suslin tree $]$.

We will also use the following consequence of a well-known lemma of Todorcevic called the pressing-down lemma for non-special trees. This more general lemma appears as Theorem 2.4 in [14]. The reader should also consult Theorems 13 and 14 of $[17]$.

Lemma 12. Let $R \subset \mathbb{S}$ be a Suslin tree. Suppose $f: R \backslash\{\min (R)\} \rightarrow R$ is a function such that $\forall x \in R \backslash\{\min (R)\}[f(x)<x]$. Then $\exists U \in[R \backslash\{\min (R)\}]^{\omega_{1}} \exists s \in R \forall x \in$ $U[f(x)=s]$.

Another useful fact about Suslin trees that is easy to verify is the following. 
Lemma 13. Let $T \subset \mathbb{S}$ be a Suslin tree. If $X \in[T]^{\omega_{1}}$, then there exists $x \in X$ such that $X$ is dense above $x$ in $T$.

We come to the main result that will be established in this section. Our claim that $\omega_{1} \rightarrow\left(\omega_{1}, \alpha\right)^{2}$ for all $\alpha<\omega_{1}$ after forcing with $\mathbb{S}$ will follow from this result. Theorem 14 gives a positive partition relation for $\mathbb{S}^{[2]}$ under $\operatorname{PFA}(\mathbb{S})$.

Theorem 14. Assume PFA(S). Let $S \in[\mathbb{S}]^{\omega_{1}}$ and $c: S^{[2]} \rightarrow 2$. Then either there exist $Y \in[S]^{\omega_{1}}$ and $g: Y \rightarrow \mathbb{S}$ such that $\forall y \in Y[g(y) \geq y]$ and $Y_{g}^{[2]} \subset K_{0}$ or for each $\alpha<\omega_{1}$, there exist $s \in S$ and $B \subset \operatorname{pred}_{S}(s)$ such that $\operatorname{otp}(B)=\alpha$ and $B^{[2]} \subset K_{1}$.

Corollary 15. $\operatorname{PFA}(\mathbb{S})$ implies that the coherent Suslin tree $\mathbb{S}$ forces $\omega_{1} \rightarrow\left(\omega_{1}, \alpha\right)^{2}$ to hold for every $\alpha<\omega_{1}$.

Proof (assuming Theorem 14). Let $\stackrel{\circ}{f} \in \mathbf{V}^{\mathbb{S}}$ be such that $\Vdash \dot{f}:\left[\omega_{1}\right]^{2} \rightarrow 2$. Fix $s \in$ $\mathbb{S}$. Suppose that $s \Vdash \neg \exists A \in\left[\omega_{1}\right]^{\omega_{1}}\left[\AA^{\prime \prime}[A]^{2}=\{0\}\right]$. We will show that $s \Vdash \forall \alpha<$ $\omega_{1} \exists A \subset \omega_{1}\left[\operatorname{otp}(A)=\alpha \wedge f^{\prime \prime}[A]^{2}=\{1\}\right]$. Fix $\alpha<\omega_{1}$ and $t \geq s$. Let $\theta$ be a sufficiently large regular cardinal and let $\left\langle M_{\xi}: \xi<\omega_{1}\right\rangle$ be an increasing continuous $\in$-chain of countable elementary submodels of $H(\theta)$, with $M_{0}$ containing all the relevant objects. For each $\xi<\omega_{1}$, put $\delta_{\xi}=M_{\xi} \cap \omega_{1}$. It is clear that for any $\xi<\omega_{1}, \beta<\gamma<\delta_{\xi}$, and $x \in \mathbb{S}$, if $\operatorname{ht}(x) \geq \delta_{\xi}$, then there exists $i \in 2$ such that $x \Vdash f(\{\beta, \gamma\})=i$. Put $S=\left\{x \in \operatorname{cone}_{\mathbb{S}}(t): \exists \xi<\omega_{1}\left[\mathrm{ht}(x)=\delta_{\xi+1}\right]\right\}$. For $x \in S$, let $\xi_{x}<\omega_{1}$ be such that $\operatorname{ht}(x)=\delta_{\xi_{x}+1}$. Define $c: S^{[2]} \rightarrow 2$ as follows. Given $x, y \in S$ with $x<y$, let $c(\{x, y\}) \in 2$ be such that $y \Vdash \stackrel{\circ}{f}\left(\left\{\delta_{\xi_{x}}, \delta_{\xi_{y}}\right\}\right)=c(\{x, y\})$. First suppose that there are $Y \in[S]^{\omega_{1}}$ and $g: Y \rightarrow \mathbb{S}$ with $\forall y \in Y[g(y) \geq y]$ and $Y_{g}^{[2]} \subset K_{0}$. Choose $x \in Y$ such that $Y$ is dense above $x$ in $\mathbb{S}$ (by applying Lemma 13). If $G$ is a $(\mathbf{V}, \mathbb{S}$ )-generic filter with $x \in G$, then in $\mathbf{V}[G]$, it is possible to find an uncountable $Z \subset G \cap Y$ such that $\forall y, z \in Z[y<z \Longrightarrow g(y) \leq z]$. Now, $\left\{\delta_{\xi_{y}}: y \in Z\right\}$ is an uncountable 0-homogeneous set for $f[G]$. As $s \in G$, this contradicts the hypothesis on $s$. So by Theorem 14, there is $x \in S$ and $B \subset \operatorname{pred}_{S}(x)$ such that $\operatorname{otp}(B)=\alpha$ and $B^{[2]} \subset K_{1}$. Then letting $A=\left\{\delta_{\xi_{b}}: b \in B\right\}$, otp $(A)=\alpha$ and $x \Vdash f^{\prime \prime}[A]^{2}=\{1\}$. As $x \geq t$, this completes the proof.

Definition 16. Let $\chi$ be a sufficiently large regular cardinal. Let $S \in[\mathbb{S}]^{\omega_{1}}$ and $c$ : $S^{[2]} \rightarrow 2$. Define a poset $\mathbb{P}(S, c)$ as follows. A condition in $\mathbb{P}(S, c)$ is a pair $p=\left\langle F_{p}, \mathcal{N}_{p}\right\rangle$ such that

(1) $F_{p} \in[S]^{<\omega}$ such that $F_{p}{ }^{[2]} \subset K_{0}$.

(2) $\mathcal{N}_{p}$ is a finite $\in$-chain of countable elementary submodels of $H(\chi)$ that contain all the relevant objects.

(3) $\forall s, t \in F_{p} \exists M \in \mathcal{N}_{p}[|M \cap\{s, t\}|=1]$.

(4) $\exists M \in \mathcal{N}_{p}\left[M \cap F_{p}=0\right]$.

For $p, q \in \mathbb{P}(S, c), q \leq p$ iff $F_{q} \supset F_{p}$ and $\mathcal{N}_{q} \supset \mathcal{N}_{p}$.

Lemma 17. Let $S \in[\mathbb{S}]^{\omega_{1}}$ and $c: S^{[2]} \rightarrow 2$. For each $\alpha<\omega_{1}$, put $D_{\alpha}=\{q \in \mathbb{P}(S, c)$ : $\left.\exists t \in F_{q}[\mathrm{ht}(t)>\alpha]\right\} . D_{\alpha}$ is a dense subset of $\mathbb{P}(S, c)$. 
Proof. Fix $\alpha<\omega_{1}$ and $p \in \mathbb{P}(S, c)$. Let $\left\{M_{0}, \ldots, M_{l}\right\}$ enumerate $\mathcal{N}_{p}$ in $\in$-increasing order. By (4) of Definition 16, $M_{0} \cap F_{p}=0$. Put $\delta=\max \left\{\alpha, M_{l} \cap \omega_{1}\right\}$. We will find $v \in S$ such that $\operatorname{ht}(v)>\delta$ and $\neg \exists s \in F_{p}[s \leq v]$. Suppose that this is not possible. Fix $T \in M_{0} \cap[S]^{\omega_{1}}$ such that $\forall u \in T[\{v \in T: v \geq u\}$ is uncountable], using Lemma 11. Suppose $u \in T \cap M_{0}$. There is a $v \in T$ so that ht $(v)>\delta$ and $v \geq u$. By the assumption, there exists $s \in F_{p}$ such that $s \leq v$. It follows that $u \leq s$. Thus $\forall u \in T \cap M_{0} \exists s \in F_{p}[u \leq s]$. It follows that $T$ is an uncountable subset of $\mathbb{S}$ with the property that $\forall A \in[T]^{\leq \omega} \exists F \in[S]^{<\omega} \forall u \in A \exists s \in F[u \leq s]$. However, it is impossible to have such an uncountable subset of a Suslin tree.

Now, fix $v \in S$ such that ht $(v)>\delta$ and $\neg \exists s \in F_{p}[s \leq v]$. Put $F_{q}=F_{p} \cup\{v\}$. Find a countable $M_{l+1} \prec H(\chi)$ with $v, p \in M_{l+1}$. Put $\mathcal{N}_{q}=\mathcal{N}_{p} \cup\left\{M_{l+1}\right\}$. Then $q \leq p$ and $q \in D_{\alpha}$.

Lemma 18. Let $\chi$ be the cardinal fixed in Definition 16. Fix $M \prec H(\chi)$ countable with $\mathbb{S} \in M$. Let $D \subset \mathbb{S}$ with $D \in M$. Let $L \subset \mathbb{S}$ be such that $\{\operatorname{ht}(x): x \in L \cap M\}$ is unbounded in $M \cap \omega_{1}$. Suppose that $\forall x \in L \cap M \exists y \in D[x \leq y]$. Then there exists $x \in L \cap M$ such that $D$ is dense above $x$ in $\mathbb{S}$. Moreover, if there exists $s \in \mathbb{S}$ such that $L=\operatorname{pred}_{\mathbb{S}}(s)$, then $\{\operatorname{ht}(x): x \in L \cap M \cap D\}$ is unbounded in $M \cap \omega_{1}$.

Proof. Put $\delta=M \cap \omega_{1}$. Put $E=\left\{x \in \mathbb{S}: \operatorname{cone}_{D}(x)=0\right\} . E \in M$. So there exists $A \in M$ such that $A \subset E, A$ is an antichain, and $A$ is maximal with respect to these two properties. As $A$ is countable, find $\alpha<\delta$ such that $A \subset \mathbb{S}_{<\alpha}$. Let $x \in L \cap M$ be such that $\operatorname{ht}(x) \geq \alpha$. If $D$ is not dense above $x$ in $\mathbb{S}$, then there is $s \in \mathbb{S}$ such that $s \geq x$ and $\operatorname{cone}_{D}(s)=0$. Thus $s \in E$ and is comparable to some $a \in A$. It follows that $a \leq x$. However, by hypothesis, there is $y \in D$ with $x \leq y$. $y \in \operatorname{cone}_{D}(a)$, contradicting $a \in E$.

For the second statement assume that $L=\operatorname{pred}_{\mathbb{S}}(s)$ for some $s \in \mathbb{S}$, and fix $\alpha<M \cap \omega_{1}$. By the first statement, fix $x \in L \cap M$ such that $D$ is dense above $x$ in $\mathbb{S}$. Note that $\operatorname{cone}_{D}(x) \in M$ and that it is an uncountable set. Put $B=\{y \in$ cone $\left._{D}(x): \operatorname{ht}(y)>\alpha\right\} \in M$. Choose $A \in M$ such that $A \subset B, A$ is an antichain, and $A$ is maximal with respect to these two properties. As $A$ is countable, fix $\beta<M \cap \omega_{1}$ such that $A \subset \mathbb{S}_{<\beta}$. Fix $t \in L \cap M$ with $h t(t)>\max \{\beta$, ht $(x)\}$. Thus $t \geq x$ and there is $y \in D$ with $y \geq t$. Since $y \in B$, there is $a \in A$ such that $a \leq y$. It follows that $a \leq t \leq s$. Therefore, $a \in L \cap D \cap M$ and ht $(a)>\alpha$.

Lemma 19. Let $T \subset \mathbb{S}$ be a Suslin tree. Suppose $c: T^{[2]} \rightarrow 2$. Either there exists $X \in[T]^{\omega_{1}}$ such that $\mathbb{P}\left(X, c \uparrow X^{[2]}\right)$ is proper and preserves $\mathbb{S}$ or for each $X \in[T]^{\omega_{1}}$ there exist $x_{0} \in X, Y \in[X]^{\omega_{1}}$, a sequence $\left\langle F_{\alpha}: \alpha<\omega_{1}\right\rangle$, and a function $g: Y \rightarrow \mathbb{S}$ such that

(1) For each $\alpha<\omega_{1}, F_{\alpha}$ is a non-empty finite subset of $X$ such that $\mathrm{ht}\left(\bigwedge F_{\alpha}\right)>\alpha$ (keep in mind that $\wedge F_{\alpha}$ may not be in $T$ ).

(2) $Y$ and $\left\{\bigwedge F_{\alpha}: \alpha<\omega_{1}\right\}$ are both dense above $x_{0}$ in $\mathbb{S}$.

(3) $\forall x \in Y[g(x) \geq x]$ and for each $\alpha<\omega_{1}$ and $s \in \operatorname{pred}_{Y}\left(\bigwedge F_{\alpha}\right) \cap \mathbb{S}_{<\alpha}$, if $g(s) \leq \bigwedge F_{\alpha}$, then $\exists t \in F_{\alpha}[c(\{s, t\})=1]$.

Proof. Fix a sufficiently large regular $\theta>\chi$. Suppose $X \in[T]^{\omega_{1}}$. For ease of notation, write $\mathbb{P}_{X}$ for $\mathbb{P}\left(X, c \uparrow X^{[2]}\right)$. If for any countable $M \prec H(\theta)$ containing all the relevant objects and any $p_{0} \in M \cap \mathbb{P}_{X}$, there exists $p \leq p_{0}$ such that 
$\forall t_{0} \in \mathbb{S}_{M \cap \omega_{1}}\left[\left\langle p, t_{0}\right\rangle\right.$ is $\left(M, \mathbb{P}_{X} \times \mathbb{S}\right)$ generic $]$, then $\mathbb{P}_{X}$ is proper and preserves $\mathbb{S}$. Assume that this fails and fix $M \prec H(\theta)$ and $p_{0} \in M \cap \mathbb{P}_{X}$ witnessing this. Put $\delta=M \cap \omega_{1}$. Put $F_{p}=F_{p_{0}}$ and $\mathcal{N}_{p}=\mathcal{N}_{p_{0}} \cup\{M \cap H(\chi)\}$. Then $p=\left\langle F_{p}, \mathcal{N}_{p}\right\rangle \in \mathbb{P}_{X}$ and extends $p_{0}$. Let $t_{0} \in \mathbb{S}_{\delta}$ and let $D \in M$ be a dense open subset of $\mathbb{P}_{X} \times \mathbb{S}$ such that $D \cap M$ is not predense below $\left\langle p, t_{0}\right\rangle$. Fix $\langle q, t\rangle \leq\left\langle p, t_{0}\right\rangle$ which is incompatible with every element of $D \cap M$. By extending it if necessary, we may assume that $\langle q, t\rangle \in D$, that $\forall N \in \mathcal{N}_{q}\left[\right.$ ht $\left.(t)>N \cap \omega_{1}\right]$, and that $\exists N \in M \cap \mathcal{N}_{q}\left[M \cap F_{q} \subset N\right]$. Put $F_{q_{0}}=F_{q} \cap M$ and $\mathcal{N}_{q_{0}}=\mathcal{N}_{q} \cap M$. It is clear that $q_{0}=\left\langle F_{q_{0}}, \mathcal{N}_{q_{0}}\right\rangle \in \mathbb{P}_{X}$ and that $q_{0} \in M$.

Let $\left\{N_{0}^{*}, \ldots, N_{k}^{*}\right\}$ enumerate $\mathcal{N}_{q} \backslash \mathcal{N}_{q_{0}}$ in $\in$-increasing order. Let $F=F_{q} \backslash F_{q_{0}}$. If $F=0$, then consider $D^{*}$, collection of all $t^{*} \in \mathbb{S}$ for which there exists $\left\{N_{0}, \ldots, N_{k}\right\}$ such that

(4) $\left\{N_{0}, \ldots, N_{k}\right\}$ is an $\in$-chain of countable elementary submodels of $H(\chi)$ containing the relevant objects, and containing $q_{0}$.

(5) $\left\langle\left\langle F_{q_{0}}, \mathcal{N}_{q_{0}} \cup\left\{N_{0}, \ldots, N_{k}\right\}\right\rangle, t^{*}\right\rangle \in D$.

$D^{*} \in M$ and it follows from Lemma 18 (applied to $\left.M \cap H(\chi)\right)$ that there exists $t^{*} \in$ $D^{*} \cap M \cap \operatorname{pred}_{\mathbb{S}}(t)$. If $\left\{N_{0}, \ldots, N_{k}\right\} \in M$ witnesses (4) and (5) for $t^{*}$, then $\left\langle\left\langle F_{q_{0}}, \mathcal{N}_{q_{0}} \cup\right.\right.$ $\left.\left.\left\{N_{0}, \ldots, N_{k}\right\}\right\rangle, t^{*}\right\rangle \in D \cap M$ and is compatible with $\langle q, t\rangle$. As this contradicts the choice of $\langle q, t\rangle$, we may assume that $F \neq 0$. Let $\left\{z_{0}, \ldots, z_{m}\right\}$ enumerate $F$ in increasing order of their heights. For each $0 \leq i, j \leq m$, put $G_{i, j}=\left\{\xi \in \operatorname{dom}\left(z_{i}\right) \cap \operatorname{dom}\left(z_{j}\right): z_{i}(\xi) \neq\right.$ $\left.z_{j}(\xi)\right\}$ and $G_{i}=\left\{\xi \in \operatorname{dom}\left(z_{i}\right) \cap \operatorname{dom}(t): t(\xi) \neq z_{i}(\xi)\right\}$. These sets are all finite. Choose $\zeta<\delta$ such that for each $0 \leq i, j \leq m, G_{i, j} \cap M \subset \zeta, G_{i} \cap M \subset \zeta$, and for each $N \in \mathcal{N}_{q_{0}}\left[N \cap \omega_{1} \in \zeta\right]$. For $0 \leq i \leq m$, put $y_{i}=z_{i} \uparrow \zeta$, and put $t^{*}=t \uparrow \zeta$. For each $0 \leq i \leq m$, choose an automorphism $\phi_{i}: \mathbb{S} \rightarrow \mathbb{S}$ such that $\phi_{i}\left(t^{*}\right)=y_{i}$ and for all $\alpha \geq \zeta$ and all $u \in \mathbb{S}_{\alpha}\left[\phi_{i}(u)=\phi_{i}(u \uparrow \zeta) \cup u \uparrow[\zeta, \alpha)\right]$. We may assume that $\phi_{i} \in M$. It is easy to see that for any $s \in M$ with $t^{*} \leq s \leq t$ and any $0 \leq i \leq m$, $y_{i} \leq \phi_{i}(s) \leq z_{i}$. Also, for any $\zeta \leq \alpha<\delta, 0 \leq i, j \leq m$, and $s \in \mathbb{S}_{\alpha} \cap \operatorname{cone}_{\mathbb{S}}\left(y_{i}\right)$, if $s \leq z_{j}$, then $s=\phi_{i}(t \uparrow \alpha)$. There are two types of points in $F$ that we must deal with. Put $I_{0}=\left\{0 \leq i \leq m: G_{i} \backslash M=0\right\}$ and $I_{1}=\left\{0 \leq i \leq m: G_{i} \backslash M \neq 0\right\}$. Observe that if $i \in I_{1}$, then $\phi_{i}\left(t \uparrow \operatorname{ht}\left(z_{i}\right)\right) \neq z_{i}$. On the other hand if $i \in I_{0}$, then $\phi_{i}\left(t \uparrow \operatorname{ht}\left(z_{i}\right)\right)=z_{i}$. For any $s \geq t^{*}$, define a two-player game $\mathscr{G}(s)$ as follows. The game lasts $m+2$ moves. In the first move, I chooses $s_{0} \geq s$ and II responds with a pair $\left\langle x_{0}, u_{0}\right\rangle$ that satisfies $s_{0} \leq x_{0} \leq u_{0}$. In the next move, I chooses $s_{1}$ with $u_{0} \leq s_{1}$. At the end of $m+2$ moves the players have constructed a sequence

$$
s_{0},\left\langle x_{0}, u_{0}\right\rangle, \ldots, s_{m+1},\left\langle x_{m+1}, u_{m+1}\right\rangle
$$

such that $s \leq s_{0} \leq x_{0} \leq u_{0} \leq s_{1} \leq x_{1} \leq u_{1} \leq \cdots \leq s_{m+1} \leq x_{m+1} \leq u_{m+1}$. We say that II wins $\mathscr{G}(s)$ if there exist $\left\{N_{0}, \ldots, N_{k}\right\}$ and $\left\{v_{i}: i \in I_{1}\right\}$ such that

(6) $\left\{N_{0}, \ldots, N_{k}\right\}$ is an $\in$-chain of countable elementary submodels of $H(\chi)$ containing the relevant objects, and containing $q_{0}$.

(7) $\forall i \in I_{1}\left[v_{i} \in \mathbb{S}_{\mathrm{ht}\left(x_{i}\right)} \cap \operatorname{cone}_{\mathbb{S}}\left(y_{i}\right)\right.$ and $\left.v_{i} \neq \phi_{i}\left(x_{i}\right)\right]$.

(8) $\left\langle\left\langle F_{q_{0}} \cup\left\{v_{i}: i \in I_{1}\right\} \cup\left\{\phi_{i}\left(x_{i}\right): i \in I_{0}\right\}, \mathcal{N}_{q_{0}} \cup\left\{N_{0}, \ldots, N_{k}\right\}\right\rangle, x_{m+1}\right\rangle \in D$.

Let $D^{*}=\left\{s \geq t^{*}\right.$ : II has a winning strategy in $\left.\mathscr{G}(s)\right\}$. Then $D^{*} \in M$. Now we have the following claim:

Claim 20. $\forall s^{*} \in M \cap \operatorname{pred}_{\mathbb{S}}(t) \exists s \in D^{*}\left[s^{*} \leq s\right]$. 
Proof. Suppose not. Fix $s^{*} \in M \cap \operatorname{pred}_{\mathbb{S}}(t)$ with $s^{*} \geq t^{*}$ such that for each $s \geq s^{*}$, I has a winning strategy in $\mathscr{G}(s)$. Fix $\Sigma \in M$ such that

$$
\forall s \geq s^{*}[\Sigma(s) \text { is a winning strategy for I in } \mathscr{G}(s)] \text {. }
$$

Consider $D_{0}=\left\{s_{0} \in \mathbb{S}: \exists s \geq s^{*}\left[s_{0}\right.\right.$ is the first move of I according to $\left.\left.\Sigma(s)\right]\right\}$. $D_{0} \in$ $M$ and applying Lemma 18, fix $s_{0} \in M \cap \operatorname{pred}_{\mathbb{S}}(t)$ and $s^{*} \leq s \leq s_{0}$ such that $s_{0}$ is the first move of I according to $\Sigma(s)$. Observe that $\Sigma(s) \in M$ and we can think of $\Sigma(s)$ as a subset of $\mathbb{S}^{<\omega} \times \mathbb{S}$. Hence $\Sigma(s) \in M \cap H(\chi)$ and hence $\Sigma(s) \in N_{i}^{*}$ for all $0 \leq i \leq k$. Now define a run of $\mathscr{G}(s)$ according to $\Sigma(s)$ as follows. Fix $0 \leq i \leq m$ and suppose that for all $j<i, s_{j}$ and $\left\langle x_{j}, u_{j}\right\rangle$ have already been specified in such a way that $s_{j}$ is according to $\Sigma(s), x_{j}=t \uparrow \mathrm{ht}\left(z_{j}\right)$, and if $s_{i}$ is the continuation of this play according to $\Sigma(s)$, then $s_{i} \in \operatorname{pred}_{\mathbb{S}}(t)$ and $\operatorname{ht}\left(s_{i}\right)<\operatorname{ht}\left(z_{i}\right)$ (when $i=0$ this is satisfied because $s_{0} \in M \cap \operatorname{pred}_{\mathbb{S}}(t)$, and since $\left.z_{0} \notin M, \operatorname{ht}\left(z_{0}\right) \geq \delta>\operatorname{ht}\left(s_{0}\right)\right)$. Let $0 \leq l \leq k$ be minimal such that $z_{i} \in N_{l}^{*}$. Note that $\left\langle s_{0},\left\langle x_{0}, u_{0}\right\rangle, \ldots, s_{i}\right\rangle \in N_{l}^{*}$, that $t \uparrow \operatorname{ht}\left(z_{i}\right) \in N_{l}^{*}$, and that $s_{i} \leq t \uparrow h t\left(z_{i}\right)$. Put $x_{i}=t \uparrow h t\left(z_{i}\right)$ and define $D_{i+1}$ as

$$
\left\{s_{i+1} \in \mathbb{S}: \exists u_{i} \geq x_{i}\left[s_{i+1} \text { is according to } \Sigma(s) \text { at } s_{0},\left\langle x_{0}, u_{0}\right\rangle, \ldots, s_{i},\left\langle x_{i}, u_{i}\right\rangle\right]\right\} .
$$

$D_{i+1} \in N_{l}^{*}$. Applying Lemma 18 choose $s_{i+1} \in N_{l}^{*} \cap \operatorname{pred}_{\mathbb{S}}(t)$ and $x_{i} \leq u_{i} \leq s_{i+1}$ such that $s_{i+1}$ is according to $\Sigma(s)$ at $s_{0},\left\langle x_{0}, u_{0}\right\rangle, \ldots, s_{i},\left\langle x_{i}, u_{i}\right\rangle$. Note that if $i+1 \leq m$, then since $z_{i+1} \notin N_{l}^{*}, \operatorname{ht}\left(z_{i+1}\right) \geq N_{l}^{*} \cap \omega_{1}>\mathrm{ht}\left(s_{i+1}\right)$, so that the construction can be continued, while if $i+1=m+1$, then $s_{i+1} \leq t$, so that $x_{m+1}=u_{m+1}=t$ is a permissible last move for II. Now, it is clear that $s_{0},\left\langle x_{0}, u_{0}\right\rangle, \ldots, s_{m+1},\left\langle x_{m+1}, u_{m+1}\right\rangle$ is a run of $\mathscr{G}(s)$ according to $\Sigma(s)$. However, if we let $N_{l}=N_{l}^{*}$, for each $0 \leq l \leq k$ and $v_{i}=z_{i}$ for all $i \in I_{1}$, then it is clear that (6)-(8) are satisfied. So II wins this run of $\mathscr{G}(s)$, contradicting that $\Sigma(s)$ is a winning strategy for I.

Using Lemma 18 and Claim 20, fix $s \in M \cap \operatorname{pred}_{\mathbb{S}}(t)$ such that $s \geq t^{*}$ and II wins $\mathscr{G}(s)$. Let $\Sigma(s) \in M$ be a winning strategy for II. For a fixed $i \in I_{0}$ consider the following statement:

if for each $j<i, s_{j}, x_{j}, u_{j} \in M \cap \operatorname{pred}_{\mathbb{S}}(t)$ are given such that they form

$\left(*_{i}\right) \quad$ a partial run of $\mathscr{G}(s)$ according to $\Sigma(s)$, then there exists a continuation

$s_{i}, x_{i}, u_{i} \in M \cap \operatorname{pred}_{\mathbb{S}}(t)$ according to $\Sigma(s)$ of this partial run such that

there is no $0 \leq l \leq m$ so that $c\left(\left\{\phi_{i}\left(x_{i}\right), z_{l}\right\}\right)$ is defined and is equal to 1 .

Assume for a moment that $\left(*_{i}\right)$ holds for all $i \in I_{0}$. Then using Lemma 18, it is possible to choose a run $s_{0},\left\langle x_{0}, u_{0}\right\rangle, \ldots, s_{m+1},\left\langle x_{m+1}, u_{m+1}\right\rangle$ of $\mathscr{G}(s)$ according to $\Sigma(s)$ such that for each $0 \leq i \leq m+1, s_{i}, x_{i}, u_{i} \in M \cap \operatorname{pred}_{\mathbb{S}}(t)$ and for each $i \in I_{0}$ and $0 \leq l \leq m$, if $c\left(\left\{\phi_{i}\left(x_{i}\right), z_{l}\right\}\right)$ is defined, then it is equal to 0 . As this run lies in $M$, choose $\left\{N_{0}, \ldots, N_{k}\right\} \in M$ and $\left\{v_{i}: i \in I_{1}\right\} \in M$ such that (6)-(8) are satisfied. Put $F_{r}=F_{q_{0}} \cup\left\{v_{i}: i \in I_{1}\right\} \cup\left\{\phi_{i}\left(x_{i}\right): i \in I_{0}\right\}$ and $\mathcal{N}_{r}=\mathcal{N}_{q_{0}} \cup$ $\left\{N_{0}, \ldots, N_{k}\right\}$. Then $r=\left\langle F_{r}, \mathcal{N}_{r}\right\rangle \in M$ and $\left\langle r, x_{m+1}\right\rangle \in M \cap D$. Moreover, note that $\forall i \in I_{1} \forall 0 \leq l \leq m\left[v_{i} \not \leq z_{l}\right]$, and that for any $i \in I_{0}$ and $0 \leq l \leq m$, if $\phi_{i}\left(x_{i}\right) \leq z_{l}$, then $c\left(\left\{\phi_{i}\left(x_{i}\right), z_{l}\right\}\right)=0$. Therefore, $\left\langle\left\langle F_{r} \cup\left\{z_{0}, \ldots, z_{m}\right\}, \mathcal{N}_{r} \cup\left\{N_{0}^{*}, \ldots, N_{k}^{*}\right\}\right\rangle, t\right\rangle$ is a common extension of $\left\langle r, x_{m+1}\right\rangle$ and $\langle q, t\rangle$. Since this contradicts the hypothesis that no member of $D \cap M$ is compatible with $\langle q, t\rangle$, there must exist some $i \in I_{0}$ for which $\left(*_{i}\right)$ fails. Fix $i \in I_{0}$ and $s_{j}, x_{j}, u_{j} \in M \cap \operatorname{pred}_{\mathbb{S}}(t)$ for $j<i$ witnessing this. Define $u$ as follows. If $i=0$, then $u=s$, else $u=u_{i-1}$. In either case, $u \in M \cap$ 
$\operatorname{pred}_{\mathbb{S}}(t)$ and $t^{*} \leq u$. Write $v=\phi_{i}(u)$ and note that $y_{i} \leq v \leq z_{i}$. Let $E=\left\{x_{i}\right.$ : $\exists s_{i} \exists u_{i}\left[s_{0},\left\langle x_{0}, u_{0}\right\rangle, \ldots, s_{i},\left\langle x_{i}, u_{i}\right\rangle\right.$ is a partial run of $\mathscr{G}(s)$ according to $\left.\left.\Sigma(s)\right]\right\}$. Define $Y$ to be $\left\{\phi_{i}\left(x_{i}\right): x_{i} \in E\right\}$. Note that $E, Y \in M$. Since $\Sigma(s)$ is winning for II, $Y \subset X$. It is easy to see that $Y$ is dense above $v$ in $\mathbb{S}$. Indeed, let $w \in \mathbb{S}$ with $w \geq v$. Then $\phi_{i}^{-1}(w) \geq u$, and so is a legitimate $i$ th move for I. Hence there exist $x_{i}, u_{i}$ such that $s_{0},\left\langle x_{0}, u_{0}\right\rangle, \ldots, \phi_{i}^{-1}(w),\left\langle x_{i}, u_{i}\right\rangle$ is a partial run of $G(s)$ according to $\Sigma(s)$. Hence $x_{i} \in E$ and $x_{i} \geq \phi_{i}^{-1}(w)$, whence $\phi_{i}\left(x_{i}\right) \in Y$ and $\phi_{i}\left(x_{i}\right) \geq w$. There is a function $g: Y \rightarrow \mathbb{S}$ in $M$ such that for all $x \in Y$, there exists $s_{i}$ such that $s_{0},\left\langle x_{0}, u_{0}\right\rangle, \ldots, s_{i},\left\langle\phi_{i}^{-1}(x), \phi_{i}^{-1}(g(x))\right\rangle$ is a partial run of $\mathscr{G}(s)$ according to $\Sigma(s)$. Clearly, $g(x) \geq x$. Now we have:

Claim 21. For each $\alpha<\omega_{1}$, there exists $F_{\alpha}$ satisfying (1) and (3) such that $\bigwedge F_{\alpha} \geq v$.

Proof. If not, then there is $\alpha \in M$ witnessing this. Fix such $\alpha \in M$. Let $F_{\alpha}=$ $\left\{z_{j} \in F: y_{i} \leq z_{j}\right\}$. It is easy to see that $\bigwedge F_{\alpha} \notin M$, and hence $\operatorname{ht}\left(\bigwedge F_{\alpha}\right) \geq \alpha$. It is also easy to see that $\bigwedge F_{\alpha} \geq v$. Now suppose that $x \in \operatorname{pred}_{Y}\left(\bigwedge F_{\alpha}\right) \cap \mathbb{S}_{<\alpha}$, and assume that $g(x) \leq \bigwedge F_{\alpha}$. As every element of $Y$ is above $y_{i}$, this implies that $\phi_{i}^{-1}(g(x)), \phi_{i}^{-1}(x) \in M \cap \operatorname{pred}_{\mathbb{S}}(t)$ and that there is $s_{i} \in M \cap \operatorname{pred}_{\mathbb{S}}(t)$ such that $s_{0},\left\langle x_{0}, u_{0}\right\rangle, \ldots, s_{i},\left\langle\phi_{i}^{-1}(x), \phi_{i}^{-1}(g(x))\right\rangle$ is a partial run of $\mathscr{G}(s)$ according to $\Sigma(s)$. By the hypothesis that $\left(*_{i}\right)$ fails, there is $z_{l} \in F$ such that $c\left(\left\{x, z_{l}\right\}\right)=1$. In particular, $y_{i} \leq x \leq z_{l}$. So $z_{l} \in F_{\alpha}$, and (3) is satisfied.

To complete the proof of the lemma, choose $F_{\alpha}$ for each $\alpha<\omega_{1}$ as in the claim. Then $Z=\left\{\bigwedge F_{\alpha}: \alpha<\omega_{1}\right\}$ is an uncountable subset of $\mathbb{S}$. Find $z \in Z$ such that $Z$ is dense above $z$ in $\mathbb{S}$. Since $z \geq v$ and $Y$ is dense above $v$ in $\mathbb{S}$, it is possible to choose $x_{0} \in Y \subset X$ with $x_{0} \geq z$. Now both $Z$ and $Y$ are dense above $x_{0}$ in $\mathbb{S}$.

Definition 22. Let $R$ be a Suslin tree and fix $c: R^{[2]} \rightarrow 2$. Fix a $C$-sequence $\left\langle c_{\alpha}: \alpha<\omega_{1}\right\rangle$ such that if $\beta>1$, then $\forall n \in \omega\left[c_{\beta}(n)>0\right]$. For $0<\beta<\omega_{1}, t \in R$, and $n \in \omega$ define $L(\beta, t, n)$ to be the set of all $A$ such that

(1) $\exists s \in R\left[s<t\right.$ and $\left.A \subset \operatorname{pred}_{R}(s)\right]$.

(2) $\operatorname{otp}(A)=\omega^{c_{\beta}(n)}$.

(3) $A^{[2]} \subset K_{1}$.

(4) $\left\{u \in\right.$ cone $\left._{R}(t): A \otimes\{u\} \subset K_{1}\right\}$ is uncountable.

Note that no member of $L(\beta, t, n)$ is empty. Next, if $B \in L(\beta, t, n+1)$ and $A \subset B$ with $\operatorname{otp}(A)=\omega^{c_{\beta}(n)}$, then $A \in L(\beta, t, n)$. Moreover, if $t \leq u, A \in L(\beta, u, n)$, and $\exists s \in R\left[s<t\right.$ and $\left.A \subset \operatorname{pred}_{R}(s)\right]$, then $A \in L(\beta, t, n)$. Also if $t \leq u, A \in L(\beta, t, n)$, and $\left\{v \in \operatorname{cone}_{R}(u): A \otimes\{v\} \subset K_{1}\right\}$ is uncountable, then $A \in L(\beta, u, n)$.

Definition 23. Fix a well ordering of $\mathcal{P}(R)$, say 4 . For $A, B \subset R$ and $t \in R$, we say that $B$ follows $A$ with respect to $t$ if $\forall a \in A \forall b \in B[a<b], A \otimes B \subset K_{1}$, and $\left\{u \in\right.$ cone $\left._{R}(t):(A \cup B) \otimes\{u\} \subset K_{1}\right\}$ is uncountable. It is clear that if $B$ follows $A$ with respect to $t$ and $C \subset B$, then $C$ follows $A$ with respect to $t$. Also, if $t \leq u$ and $B$ follows $A$ with respect to $u$, then $B$ follows $A$ with respect to $t$. If $t \leq u, B$ follows $A$ with respect to $t$, and $\left\{v \in \operatorname{cone}_{R}(u):(A \cup B) \otimes\{v\} \subset K_{1}\right\}$ is uncountable, then $B$ follows $A$ with respect to $u$. For $t \in R$ and $0<\beta<\omega_{1}$, define a function $\sigma_{\beta, t}: \omega \rightarrow \mathcal{P}(R)$ as follows. Fix $n \in \omega$ and suppose that for all $m<n, \sigma_{\beta, t}(m)$ has 
been defined. Put $A_{\beta, t, n}=\bigcup_{m<n} \sigma_{\beta, t}(m) \subset R$. Consider

$$
\left\{B \in L(\beta, t, n): B \text { follows } A_{\beta, t, n} \text { with respect to } t\right\} .
$$

If this set is empty, then set $\sigma_{\beta, t}(n)=0$. Otherwise, set $\sigma_{\beta, t}(n)$ to be the ४-least element of this set. Define $A_{\beta, t}=\bigcup_{n \in \omega} \sigma_{\beta, t}(n)$.

It is clear that for each $n \in \omega$, either $\sigma_{\beta, t}(n)=0$ or $\sigma_{\beta, t}(n) \in L(\beta, t, n)$, but not both. In either case, observe that $\sigma_{\beta, t}(n) \subset \operatorname{pred}_{R}(t)$, that $\forall a \in A_{\beta, t, n} \forall b \in$ $\sigma_{\beta, t}(n)[a<b]$, and that $\left(A_{\beta, t, n} \otimes \sigma_{\beta, t}(n)\right) \cup\left(\sigma_{\beta, t}(n)\right)^{[2]} \subset K_{1}$. Therefore, if $\forall n \in$ $\omega\left[\sigma_{\beta, t}(n) \in L(\beta, t, n)\right]$, then $A_{\beta, t}$ is a subset of $\operatorname{pred}_{R}(t)$ of order type $\omega^{\beta}$ such that $A_{\beta, t}^{[2]} \subset K_{1}$. Furthermore, for all $t \in R \backslash\{\min (R)\}$ and all $n \in \omega$, there is $s \in R$ with $s<t$ such that $A_{\beta, t, n} \subset \operatorname{pred}_{R}(s)$.

Lemma 24. For any $t \in R$ and $0<\beta<\omega_{1}$, if there exists $m \in \omega$ such that $\sigma_{\beta, t}(m)=0$, then $\forall n \geq m\left[\sigma_{\beta, t}(n)=0\right]$.

Proof. Prove by induction on $n \geq m$ that $\sigma_{\beta, t}(n)=0$. When $n=m$, this is the hypothesis. Suppose this is true for $n \geq m$. Then $A_{\beta, t, n+1}=A_{\beta, t, n} \cup \sigma_{\beta, t}(n)=A_{\beta, t, n}$. If $\sigma_{\beta, t}(n+1) \neq 0$, then $\sigma_{\beta, t}(n+1) \in L(\beta, t, n+1)$, and $\sigma_{\beta, t}(n+1)$ follows $A_{\beta, t, n}$ with respect to $t$. In particular, $\operatorname{otp}\left(\sigma_{\beta, t}(n+1)\right)=\omega^{c_{\beta}(n+1)}$. Choose $B \subset \sigma_{\beta, t}(n+1)$ with $\operatorname{otp}(B)=\omega^{c_{\beta}(n)}$. Then $B \in L(\beta, t, n)$ and $B$ follows $A_{\beta, t, n}$ with respect to $t$. This contradicts the fact that $\sigma_{\beta, t}(n)=0$.

Definition 25. For $t \in R$ and $0<\beta<\omega_{1}$, if there is $n \in \omega$ such that $\sigma_{\beta, t}(n)=0$, then let $n_{\beta, t}$ be the least such $n$. Otherwise, put $n_{\beta, t}=\omega$. Define $X_{\beta, t}=\{u \in$ cone $\left._{R}(t): A_{\beta, t} \otimes\{u\} \subset K_{1}\right\}$.

Note that $A_{\beta, t}=\bigcup_{n<n_{\beta, t}} \sigma_{\beta, t}(n)$. Observe also that if $n_{\beta, t}<\omega$, then $X_{\beta, t}$ is uncountable.

Lemma 26. Fix $t, u \in R$ with $t \leq u$, and $0<\beta<\omega_{1}$. Suppose that $X_{\beta, t} \cap \operatorname{cone}_{R}(u)$ is uncountable. Moreover assume that $\exists s \in R\left[s<t\right.$ and $\left.A_{\beta, u} \subset \operatorname{pred}_{R}(s)\right]$. Then $\sigma_{\beta, t}=$ $\sigma_{\beta, u}$.

Proof. First a preliminary observation: it follows from the hypotheses that for each $n \in \omega,\left\{v \in \operatorname{cone}_{R}(u): \sigma_{\beta, t}(n) \otimes\{v\} \subset K_{1}\right\}$ and $\left\{v \in \operatorname{cone}_{R}(u): A_{\beta, t, n} \otimes\{v\} \subset K_{1}\right\}$ are both uncountable. Now suppose for a contradiction that there exists $n \in \omega$ such that $\sigma_{\beta, t}(n) \neq \sigma_{\beta, u}(n)$, and choose the minimal $n \in \omega$ with this property. Then $A_{\beta, t, n}=A_{\beta, u, n}$. Assume that $\sigma_{\beta, t}(n) \neq 0$. Thus $\sigma_{\beta, t}(n) \in L(\beta, t, n)$ and $\sigma_{\beta, t}(n)$ follows $A_{\beta, t, n}$ with respect to $t$. Since $\left\{v \in \operatorname{cone}_{R}(u): \sigma_{\beta, t}(n) \otimes\{v\} \subset K_{1}\right\}$ is uncountable, $\sigma_{\beta, t}(n) \in L(\beta, u, n)$. Also, since $\left\{v \in \operatorname{cone}_{R}(u):\left(A_{\beta, t, n} \cup \sigma_{\beta, t}(n)\right) \otimes\{v\} \subset\right.$ $\left.K_{1}\right\}=\left\{v \in \operatorname{cone}_{R}(u): A_{\beta, t, n+1} \otimes\{v\} \subset K_{1}\right\}$ is uncountable, $\sigma_{\beta, t}(n)$ follows $A_{\beta, u, n}$ with respect to $u$. It follows that $\sigma_{\beta, u}(n) \neq 0$ and $\sigma_{\beta, u}(n) \triangleleft \sigma_{\beta, t}(n)$.

Next suppose that $\sigma_{\beta, u}(n) \neq 0$. Then $\sigma_{\beta, u}(n) \in L(\beta, u, n)$ and $\sigma_{\beta, u}(n)$ follows $A_{\beta, u, n}$ with respect to $u$. Note that there is $s \in R$ such that $s<t$ and $\sigma_{\beta, u}(n) \subset$ $A_{\beta, u} \subset \operatorname{pred}_{R}(s)$. Therefore, $\sigma_{\beta, u}(n) \in L(\beta, t, n)$. Also, $\sigma_{\beta, u}(n)$ follows $A_{\beta, t, n}$ with respect to $t$. Thus $\sigma_{\beta, t}(n) \neq 0$ and $\sigma_{\beta, t}(n) \sigma_{\beta, u}(n)$. However, these two implication that we have established imply that both $\sigma_{\beta, t}(n)$ and $\sigma_{\beta, u}(n)$ are equal to 0 , a contradiction. 
Next is a lemma that is of independent interest and can be considered as a part of set theory folklore. It asserts the existence of certain types of ultrafilters on countable indecomposable ordinals under the hypothesis $\mathfrak{p}>\omega_{1}$. This lemma also plays a similar role in Todorcevic's proof that PID $+\mathfrak{p}>\omega_{1}$ implies $\omega_{1} \rightarrow\left(\omega_{1}, \alpha\right)^{2}$.

Lemma 27. Assume $\mathfrak{p}>\omega_{1}$. For each $0<\beta<\omega_{1}$ and a well ordered set $X=$ $\left\langle X,<_{X}\right\rangle$ of order type $\omega^{\beta}$, there is an ultrafilter $\mathcal{U}_{\beta}(X)$ on $X$ such that

(1) For each $A \in \mathcal{U}_{\beta}(X) \operatorname{otp}(A)=\omega^{\beta}$.

(2) For any $\mathcal{F} \subset \mathcal{U}_{\beta}(X)$ of size at most $\omega_{1}$, there is $Y \subset X$ such that $\operatorname{otp}(Y)=\omega^{\beta}$ and $\forall Z \in \mathcal{F}[Y \backslash Z$ is a bounded subset of $X]$. Moreover if $|\mathcal{F}|=\omega_{1}$, then there exists $\mathcal{G} \in[\mathcal{F}]^{\omega_{1}}$ such that $\operatorname{otp}(\bigcap \mathcal{G})=\omega^{\beta}$.

Proof. The proof is by induction on $\beta$. If $\beta=1$, then $X$ has order type $\omega$, and we can let $\mathcal{U}_{\beta}(X)$ be any ultrafilter on $X$. It is clear that (1) is satisfied. For (2), fix $\mathcal{F} \subset \mathcal{U}_{\beta}(X)$ with $|\mathcal{F}| \leq \omega_{1}$. As $\mathfrak{p}>\omega_{1}$, there is $Y \in[X]^{\omega}$ such that $\forall Z \in \mathcal{F}[Y \backslash Z$ is finite]. It is clear that $\operatorname{otp}(Y)=\omega$ and $\forall Z \in \mathcal{F}[Y \backslash Z$ is bounded in $X]$. Next, if $|\mathcal{F}|=\omega_{1}$ and $Y \subset X$ is as above, then there is a finite $F \in[Y]^{<\omega}$ and $\mathcal{G} \in[\mathcal{F}]^{\omega_{1}}$ such that $\forall Z \in \mathcal{G}[Y \backslash F \subset Z]$. As otp $(Y \backslash F)=\omega$, it is clear that $\operatorname{otp}(\bigcap \mathcal{G})=\omega$.

Next suppose $\beta>1$. Fix $\left\langle X_{n}: n \in \omega\right\rangle$ such that $X=\bigcup_{n \in \omega} X_{n}$ and $\forall n \in$ $\omega\left[\operatorname{otp}\left(X_{n}\right)=\omega^{c_{\beta}(n)} \wedge X_{n}<_{X} X_{n+1}\right]$. Here $X_{n}<_{X} X_{n+1}$ means $\forall x \in X_{n} \forall y \in$ $X_{n+1}\left[x<_{X} y\right]$. Assume that $\mathcal{U}_{c_{\beta}(n)}\left(X_{n}\right)$ has been constructed for all $n \in \omega$. Put $\mathcal{U}_{\beta}(X)=\left\{A \subset X:\left\{n \in \omega: A \cap X_{n} \in \mathcal{U}_{c_{\beta}(n)}\left(X_{n}\right)\right\} \in \mathcal{U}_{1}(\omega)\right\}$. This is clearly an ultrafilter on $X$. It is also clear that (1) is satisfied by $\mathcal{U}_{\beta}(X)$. For (2), fix $\mathcal{F} \subset \mathcal{U}_{\beta}(X)$ of size at most $\omega_{1}$. For each $n \in \omega$, put

$$
\mathcal{F}_{n}=\left\{Z \cap X_{n}: Z \in \mathcal{F} \wedge Z \cap X_{n} \in \mathcal{U}_{c_{\beta}(n)}\left(X_{n}\right)\right\} .
$$

$\mathcal{F}_{n} \subset \mathcal{U}_{c_{\beta}(n)}\left(X_{n}\right)$ and $\left|\mathcal{F}_{n}\right| \leq \omega_{1}$. So choose $Y_{n} \subset X_{n}$ such that $\operatorname{otp}\left(Y_{n}\right)=\omega^{c_{\beta}(n)}$ and $\forall A \in \mathcal{F}_{n}\left[Y_{n} \backslash A\right.$ is a bounded subset of $\left.X_{n}\right]$. For each $Z \in \mathcal{F}$ define a function $S_{Z}$ with domain $\omega$ as follows. Given $n \in \omega$, if $Z \cap X_{n} \in \mathcal{U}_{c_{\beta}(n)}\left(X_{n}\right)$, then $S_{Z}(n)=Y_{n} \backslash\left(Z \cap X_{n}\right)$. Otherwise $S_{Z}(n)=0$. In either case, $S_{Z}(n)$ is a bounded subset of $X_{n}$. Use the fact that $\mathfrak{b}<\omega_{1}$ to choose $\left\langle B_{n}: n \in \omega\right\rangle$ such that for each $\forall n \in \omega\left[B_{n}\right.$ is a bounded subset of $\left.X_{n}\right]$ and $\forall Z \in \mathcal{F} \forall^{\infty} n \in \omega\left[S_{Z}(n) \subset B_{n}\right]$. Note that $\operatorname{otp}\left(Y_{n} \backslash B_{n}\right)=\omega^{c_{\beta}(n)}$, for all $n \in \omega$. For each $Z \in \mathcal{F}$, put $\operatorname{dom}(Z)=\{n \in \omega$ : $\left.Z \cap X_{n} \in \mathcal{U}_{c_{\beta}(n)}\left(X_{n}\right)\right\} .\{\operatorname{dom}(Z): Z \in \mathcal{F}\}$ is a subset of $\mathcal{U}_{1}(\omega)$ of size at most $\omega_{1}$. Choose $D \in[\omega]^{\omega}$ such that $\forall Z \in \mathcal{F}\left[D \backslash \operatorname{dom}(Z)\right.$ is finite]. Put $Y=\bigcup_{n \in D}\left(Y_{n} \backslash B_{n}\right)$. It is clear that $Y \subset X$ and $\operatorname{otp}(Y)=\omega^{\beta}$. Fix $Z \in \mathcal{F}$ and fix $n_{Z} \in \omega$ such that $D \backslash n_{Z} \subset \operatorname{dom}(Z)$ and $\forall n \geq n_{Z}\left[S_{Z}(n) \subset B_{n}\right]$. We claim that $Y \backslash Z$ is bounded by $\min \left(X_{n_{Z}}\right)$. Indeed fix $y \in Y \backslash Z$. Then $y \in Y_{n} \backslash B_{n}$ for some $n \in D$. If $n \geq n_{Z}$, then $n \in \operatorname{dom}(Z)$ and $S_{Z}(n) \subset B_{n}$. So $Z \cap X_{n} \in \mathcal{U}_{c_{\beta}(n)}\left(X_{n}\right)$, and so $S_{Z}(n)=Y_{n} \backslash\left(Z \cap X_{n}\right)$. As $y \in Y_{n}$ and $y \notin Z$, we have that $y \in S_{Z}(n)$. However, since $y \notin B_{n}$, this contradicts $S_{Z}(n) \subset B_{n}$. Therefore, $n<n_{z}$ and since $y \in Y_{n} \subset X_{n}$, we conclude that $y<_{X} \min \left(X_{n_{Z}}\right)$.

Now, if $|\mathcal{F}|=\omega_{1}$, then there exist $n \in \omega$ and $\mathcal{G} \in[\mathcal{F}]^{\omega_{1}}$ such that $\forall Z \in$ $\mathcal{G}\left[n_{Z}=n\right]$. Note that otp $\left(\left\{y \in Y: y<_{X} \min \left(X_{n}\right)\right\}\right)<\omega^{\beta}$. Therefore, $Y^{*}=Y \backslash$ $\left\{y \in Y: y<_{X} \min \left(X_{n}\right)\right\}$ has order type $\omega^{\beta}$. By what has been proved above, $Y^{*} \subset$ $\bigcap \mathcal{G}$, whence otp $(\bigcap \mathcal{G})=\omega^{\beta}$.

We can now finish the proof of Theorem 14 . 
Proof of Theorem 14. We show by induction on $\beta<\omega_{1}$ that for any Suslin tree $T \subset \mathbb{S}$ and any $c: T^{[2]} \rightarrow 2$, either there exists $Y \in[T]^{\omega_{1}}$ and $g: Y \rightarrow \mathbb{S}$ such that $\forall y \in$ $Y[g(y) \geq y]$ and $Y_{g}^{[2]} \subset K_{0, c}$, or for each $X \in[T]^{\omega_{1}}$ there exist $x \in X, B \subset \operatorname{pred}_{X}(x)$, and $Z \in\left[\operatorname{cone}_{X}(x)\right]^{\omega_{1}}$ such that $\operatorname{otp}(B)=\omega^{\beta}$ and $B^{[2]} \cup(B \otimes Z) \subset K_{1, c}$. This is sufficient to imply Theorem 14 , for given $S \in[\mathbb{S}]^{\omega_{1}}$ and $c: S^{[2]} \rightarrow 2$, let $T \in[S]^{\omega_{1}}$ be a Suslin tree (we are using Lemma 11). Suppose the first alternative of Theorem 14 fails and let $\alpha<\omega_{1}$ be given. Choose $\beta<\omega_{1}$ such that $\alpha \leq \omega^{\beta}$. Applying the above statement to $\beta, T$, and $c \uparrow T^{[2]}$ with $X=T$, we can get $x \in T$ and $B \subset \operatorname{pred}_{S}(x)$ such that $\operatorname{otp}(B)=\omega^{\beta}$ and $B^{[2]} \subset K_{1, c}$. Taking $B^{*} \subset B$ with $\operatorname{otp}\left(B^{*}\right)=\alpha$, we get what we want.

To prove the above statement, fix $\beta<\omega_{1}$ and assume that the statement holds for all smaller ordinals. Let $T$ and $c$ be given and suppose that the first alternative of the statement fails. In particular, this implies that there is no $X \in[T]^{\omega_{1}}$, such that $\mathbb{P}\left(X, c\left\lceil X^{[2]}\right)\right.$ is proper and preserves $\mathbb{S}$. For if not, let

$$
D_{\alpha}=\left\{q \in \mathbb{P}\left(X, c \uparrow X^{[2]}\right): \exists t \in F_{q}[\mathrm{ht}(t)>\alpha]\right\} .
$$

By Lemma $17, D_{\alpha}$ is dense in $\mathbb{P}\left(X, c \uparrow X^{[2]}\right)$. Applying PFA(S), let $G$ be a filter on $\mathbb{P}\left(X, c \uparrow X^{[2]}\right)$ such that $\forall \alpha<\omega_{1}\left[G \cap D_{\alpha} \neq 0\right]$. Let $Y=\bigcup_{q \in G} F_{q}$. Then $Y \in[T]^{\omega_{1}}$. If we let $g: Y \rightarrow \mathbb{S}$ be defined by $g(y)=y$, for all $y \in Y$, then $Y_{g}^{[2]}=Y^{[2]} \subset K_{0, c}$, contradicting the hypothesis that the first alternative of the statement fails.

Now, fix any $X \in[T]^{\omega_{1}}$. By Lemma 19 there exist $x_{0} \in X, Y \in[X]^{\omega_{1}}$, a sequence $\left\langle F_{\alpha}: \alpha<\omega_{1}\right\rangle$, and a function $g: Y \rightarrow \mathbb{S}$ satisfying (1)-(3) of Lemma 19. If $\beta=0$, then fix $y \in Y$ with $y \geq x_{0}$, and put $x=y$ and $B=\{y\}$. Then for each $\alpha<\omega_{1}$, if $\operatorname{ht}(y)<\alpha$ and $\bigwedge F_{\alpha} \geq g(y)$, then there is $t_{\alpha} \in F_{\alpha}$ such that $c\left(\left\{y, t_{\alpha}\right\}\right)=1$. Letting $Z=\left\{t_{\alpha}: \alpha>\operatorname{ht}(y)\right.$ and $\left.\bigwedge F_{\alpha} \geq g(y)\right\}$, we get what we want.

Assume now that $0<\beta<\omega_{1}$. Let $\bar{Y}=g^{\prime \prime} Y$. By Lemma 11, fix $R \subset \operatorname{cone}_{\bar{Y}}\left(x_{0}\right)$, a Suslin tree. For each $s \in R$ choose $y_{s} \in Y$ such that $g\left(y_{s}\right)=s$. Observe that if $s<t$, then $y_{s}$ and $y_{t}$ are comparable and different. Define $d: R^{[2]} \rightarrow 2$ by $d(\{s, t\})=1$ iff $y_{s}<y_{t}$ and $c\left(\left\{y_{s}, y_{t}\right\}\right)=1$, for any $s, t \in R$ with $s<t$. We claim that it is enough to find $u \in R$ and $\bar{B} \subset \operatorname{pred}_{R}(u)$ with $\operatorname{otp}(\bar{B})=\omega^{\beta}$ such that $\bar{B}^{[2]} \subset K_{1, d}$. Indeed, suppose this can be done. Choose any $x \in Y$ with $x \geq u$. Let $B^{*}=\left\{y_{s}: s \in \bar{B}\right\}$. For any $s, t \in \bar{B}$ with $s<t, y_{s}<y_{t}$ because $d(\{s, t\})=1$. So $\operatorname{otp}\left(B^{*}\right)=\omega^{\beta}$. Also it is clear that $B^{*} \subset \operatorname{pred}_{X}(x)$. If $\alpha<\omega_{1}$ is such that $\alpha>\operatorname{ht}(x)$ and $\bigwedge F_{\alpha} \geq x$, then for any $y \in B^{*}, g(y) \leq \bigwedge F_{\alpha}$, and so there is $t \in F_{\alpha}$ such that $c(\{y, t\})=1$. Therefore, letting $\mathcal{U}_{\beta}\left(B^{*}\right)$ be as in Lemma 27 (note that PFA(S) implies $\mathfrak{p}>\omega_{1}$; so Lemma 27 may be applied) and letting $I=\left\{\alpha<\omega_{1}: \alpha>\operatorname{ht}(x)\right.$ and $\left.\bigwedge F_{\alpha} \geq x\right\}$, for each $\alpha \in I$, there is $Y_{\alpha} \in \mathcal{U}_{\beta}\left(B^{*}\right)$ and $t_{\alpha} \in F_{\alpha}$ such that $\forall y \in Y_{\alpha}\left[c\left(\left\{y, t_{\alpha}\right\}\right)=1\right]$. There exists $J \in[I]^{\omega_{1}}$ such that otp $\left(\bigcap_{\alpha \in J} Y_{\alpha}\right)=\omega^{\beta}$. It is clear that $B=\bigcap_{\alpha \in J} Y_{\alpha}$, $Z=\left\{t_{\alpha}: \alpha \in J\right\}$, and $x$ are as needed.

Thus we may concentrate on finding $u \in R$ and $\bar{B} \subset \operatorname{pred}_{R}(u)$ as above. We will apply the notation of Definitions 22, 23, and 25, and Lemmas 26 and 24 to $R, d$, and $\beta$. If there exists $u \in R$ such that $n_{\beta, u}=\omega$, then letting $\bar{B}=A_{\beta, u}$ works. Thus assume that for each $u \in R, n_{\beta, u}<\omega$. Then for each $u \in R \backslash\{\min (R)\}$, there is $f(u) \in R$ such that $f(u)<u$ and $A_{\beta, u} \subset \operatorname{pred}_{R}(f(u))$. So by Lemma 12 and the pigeonhole principle there exist $U \in[R \backslash\{\min (R)\}]^{\omega_{1}}, s \in R$, and $n \in \omega$ such that $\forall u \in U\left[f(u)=s\right.$ and $\left.n_{\beta, u}=n\right]$. Fix $x \in U$ such that $U$ is dense above $x$ in $\mathbb{S}$. Since 
$n_{\beta, x}<\omega, X_{\beta, x}$ is uncountable. Choose $u \in X_{\beta, x}$ such that $X_{\beta, x}$ is dense above $u$ in $\mathbb{S}$. Apply the inductive hypothesis to $c_{\beta}(n), R$, and $d$. Suppose that the first alternative holds. Let $F \in[R]^{\omega_{1}}$ and $g^{*}: F \rightarrow \mathbb{S}$ be such that $\forall t \in F\left[g^{*}(t) \geq t\right]$ and $F_{g^{*}}^{[2]} \subset K_{0, d}$. Let $Y^{*}=\left\{y_{t}: t \in F\right\}$ and define $h: Y^{*} \rightarrow \mathbb{S}$ by $h(y)=g^{*}(g(y))$, for each $y \in Y^{*}$. Then $Y^{*} \in[T]^{\omega_{1}}, \forall y \in Y^{*}[h(y) \geq y]$, and $Y_{h}^{*[2]} \subset K_{0, c}$. This contradicts the hypothesis that the first alternative fails for $\beta, T$, and $c$. So the second alternative must hold for $c_{\beta}(n), R$, and $d$. Since $V=$ cone $_{X_{\beta, x}}(u) \in[R]^{\omega_{1}}$, we can find $v \in V, B \subset \operatorname{pred}_{V}(v)$, and $W \in\left[\operatorname{cone}_{V}(v)\right]^{\omega_{1}}$ such that $\operatorname{otp}(B)=\omega^{c_{\beta}(n)}$, and $B^{[2]} \cup(B \otimes W) \subset K_{1, d}$. Fix $w \in W$ such that $W$ is dense above $w$ in $\mathbb{S}$. Choose $y \in U$ with $y>w$. Note that $X_{\beta, x} \cap \operatorname{cone}_{R}(y)$ is uncountable. Furthermore, as $y \in U$ $f(y)=s<x$ and $A_{\beta, y} \subset \operatorname{pred}_{R}(s)$. Therefore, Lemma 26 applies and implies that $\sigma_{\beta, x}=\sigma_{\beta, y}$. In particular, $A_{\beta, x}=A_{\beta, y}$. Also, since $y \in U, n_{\beta, y}=n$. So $A_{\beta, y}=A_{\beta, y, n}$ and $\sigma_{\beta, y}(n)=0$. However, cone $_{W}(y)$ is uncountable and cone $W(y) \subset\left\{z \in\right.$ cone $_{R}(y)$ : $\left.\left(A_{\beta, y, n} \cup B\right) \otimes\{z\} \subset K_{1, d}\right\} \subset\left\{z \in \operatorname{cone}_{R}(y): B \otimes\{z\} \subset K_{1, d}\right\}$. So it is easy to check that $B \in L(\beta, y, n)$ and that $B$ follows $A_{\beta, y, n}$ with respect to $y$. However, this contradicts $\sigma_{\beta, y}(n)=0$, finishing the proof.

The reader may conjecture that the stronger form of Theorem 14 in which $g$ is always equal to the identity function holds. However, our next counterexample shows that this is provably false in ZFC. This same negative partition relation for non-special trees of cardinality $\mathfrak{c}$ with no uncountable chains and with the property that every subset of size $<\mathfrak{c}$ is special was proved under the hypothesis that $\mathfrak{p}=\mathfrak{c}$ by Todorčević in [17]. However, our result below is the first such negative partition relation known to be provable in ZFC.

Theorem 28. There is $c: \mathbb{S}^{[2]} \rightarrow 2$ such that

(1) There is no $X \in[\mathbb{S}]^{\omega_{1}}$ such that $X^{[2]} \subset K_{0}$.

(2) There is no $s \in \mathbb{S}$ and $B \subset \operatorname{pred}_{\mathbb{S}}(s)$ such that $\operatorname{otp}(B)=\omega+2$ and $B^{[2]} \subset K_{1}$.

Proof. Let $\omega^{\uparrow \omega}$ denote $\left\{f \in \omega^{\omega}: \forall n \in \omega[f(n)<f(n+1)]\right\}$. For $f, g \in \omega^{\omega}$, if $f \neq g$, let $\Delta(f, g)$ denote the least $n \in \omega$ such that $f(n) \neq g(n)$. Choose a collection $\left\{f_{s}: s \in \mathbb{S}\right\}$ of $\aleph_{1}$-many pairwise distinct elements of $\omega^{\uparrow \omega}$. For each $s \in \mathbb{S}$, let $\left\{s_{n}^{+}: n \in \omega\right\}$ be a 1-1 enumeration of $\operatorname{succ}_{\mathbb{S}}(s)$. Recall that for each $s \in \mathbb{S}, \exists^{\infty} n \in \omega\left[s^{\frown}\langle n\rangle \in \mathbb{S}\right]$. Now, define $c: \mathbb{S}^{[2]} \rightarrow 2$ as follows. For any pair $s, t \in \mathbb{S}$, if $s<t$, then there is a unique $n \in \omega$ such that $s_{n}^{+} \leq t$. If $f_{t}\left(\Delta\left(f_{s}, f_{t}\right)\right)=n$, then set $c(\{s, t\})=1$. Otherwise $c(\{s, t\})=0$. The first claim will establish (1).

Claim 29. There is no $X \in[\mathbb{S}]^{\omega_{1}}$ such that $X^{[2]} \subset K_{0}$.

Proof. It is possible to deduce this claim from Lemma 5.3 of [18]. However, we will give a self-contained proof below.

Suppose not. Fix a counterexample $X$. Let $\chi$ be a sufficiently large regular cardinal ( $\chi$ could be the cardinal fixed in Definition 16). Let $M \prec H(\chi)$ be countable with $\mathbb{S},\left\langle f_{s}: s \in \mathbb{S}\right\rangle,\left\langle\left\langle s_{n}^{+}: n \in \omega\right\rangle: s \in \mathbb{S}\right\rangle, c, X \in M$. Applying Lemma 13, let $x \in X \cap M$ be such that $X$ is dense above $x$ in $\mathbb{S}$. Fix $t \in X \backslash M$ with $x<t$, and fix $u \in X$ with $u>t$. We will get a contradiction if we can show that $f_{t}=f_{u}$. To this end, fix $m \in \omega$ and assume that $f_{t}(i)=f_{u}(i)$, for all $i<m$. Let $\sigma=f_{t} \uparrow m=f_{u} \uparrow m$. Consider any $s \in \operatorname{pred}_{X}(t) \cap M$. Let $n(s)$ denote the unique $n \in \omega$ such that $s_{n}^{+} \leq t$. If $\sigma=f_{s} \uparrow m$ 
and if $n(s)=f_{t}(m)$, then since $c(\{s, t\})=0$, it follows that $f_{s}(m)=f_{t}(m)$. Put $n=f_{t}(m)$ and $n^{*}=f_{u}(m)$. Let

$$
D=\left\{v \in \mathbb{S}: \exists s \in X\left[f_{s} \uparrow m=\sigma \text { and } f_{s}(m)=n^{*} \text { and } s_{n}^{+}=v\right]\right\} .
$$

It is easy to see that $D \in M$. Let $L=\operatorname{pred}_{\mathbb{S}}(t)$. Note that $u_{n}^{+} \in D$. Therefore, $\forall y \in L \cap M \exists v \in D[y \leq v]$. So by Lemma 18, we can find $v \in L \cap M \cap D$. Let $s \in X$ be such that $f_{s} \uparrow m=\sigma, f_{s}(m)=n^{*}$ and $s_{n}^{+}=v$. Then $s<t$ and $s \in M \cap \operatorname{pred}_{X}(t)$. Since $s_{n}^{+}=v \leq t, n(s)=n=f_{t}(m)$. Thus $f_{u}(m)=n^{*}=f_{s}(m)=f_{t}(m)$. So by induction on $m \in \omega, \forall m \in \omega\left[f_{u}(m)=f_{t}(m)\right]$, which is a contradiction.

We next work toward showing that (2) holds. We need a few preliminary claims. Aiming for a contradiction, fix $s \in \mathbb{S}$ and $B \subset \operatorname{pred}_{\mathbb{S}}(s)$ such that $\operatorname{otp}(B)=\omega+2$ and $B^{[2]} \subset K_{1}$. For each $i<\omega+2$, let $s(i)$ denote the $i$ th element of $B$, and for each $i<\omega+1$, let $n(i)$ be the unique $n \in \omega$ such that $(s(i))_{n}^{+} \in \operatorname{pred}_{\mathbb{S}}(s)$.

Claim 30. There are no infinite $A \subset \omega$ and $n \in \omega$ such that $\forall i \in A[n(i)=n]$.

Proof. Suppose not. Fix $i, j \in A$ such that $s(i)<s(j)$. Then since $c(\{s(i), s(j)\})=1$, $f_{s(j)}\left(\Delta\left(f_{s(i)}, f_{s(j)}\right)\right)=n(i)=n$. As $f_{s(j)}$ is strictly increasing, $\Delta\left(f_{s(i)}, f_{s(j)}\right) \leq n$. So it is possible to find $i, j, k \in A$ and $m \leq n$ such that $s(i)<s(j)<s(k)$ and

$$
\Delta\left(f_{s(i)}, f_{s(j)}\right)=\Delta\left(f_{s(i)}, f_{s(k)}\right)=\Delta\left(f_{s(j)}, f_{s(k)}\right)=m .
$$

However, we now have $f_{s(i)} \uparrow m=f_{s(j)} \uparrow m=f_{s(k)} \uparrow m$, and also that $f_{s(j)}(m)=$ $f_{s(k)}(m)=n$, which is impossible.

Let $\operatorname{cl}(B)$ denote the closure (with respect to the usual topology on $\omega^{\omega}$ ) of the set $\left\{f_{s(i)}: i \in \omega\right\}$. As $\operatorname{cl}(B)$ is a non-empty closed subset of $\omega^{\omega}$, fix a non-empty pruned subtree $T \subset \omega^{<\omega}$ such that $[T]=\operatorname{cl}(B)$ (refer to Section 2 for our notation for subtrees of $\left.\omega^{<\omega}\right)$. Let $\sigma \in T$. Suppose for a moment that $\exists^{\infty} n \in \omega[\sigma \frown\langle n\rangle \in T]$. Let $\operatorname{succ}_{T}(\sigma)$ denote $\{n \in \omega: \sigma \frown\langle n\rangle \in T\}$ and let $l$ denote $|\sigma|$. For each $n \in \operatorname{succ}_{T}(\sigma)$, $\operatorname{choose}$ $i_{n} \in \omega$ such that $\sigma \frown\langle n\rangle \subset f_{s\left(i_{n}\right)}$. As $B$ is well ordered, by Ramsey's theorem there is $N \in\left[\operatorname{succ}_{T}(\sigma)\right]^{\omega}$ such that $\forall m, n \in N\left[m<n \Longrightarrow s\left(i_{m}\right)<s\left(i_{n}\right)\right]$. Fix $m, n \in N$ such that $\max \left\{m, n\left(i_{m}\right)\right\}<n$. However, $\Delta\left(f_{s\left(i_{m}\right)}, f_{s\left(i_{n}\right)}\right)=l$ and $f_{s\left(i_{n}\right)}(l)=n>n\left(i_{m}\right)$, contradicting $c\left(\left\{s\left(i_{m}\right), s\left(i_{n}\right)\right\}\right)=1$. So we conclude that $T$ is finitely branching, and that $\operatorname{cl}(B)$ is a compact subset of $\omega^{\omega}$.

Now, $\left\{f_{s(i)}: i \in \omega\right\}$ is an infinite subset of $\operatorname{cl}(B)$. Let $f \in \operatorname{cl}(B)$ be a complete accumulation point of $\left\{f_{s(i)}: i \in \omega\right\}$. By applying Claim 30 and Ramsey's theorem, it is possible to choose $A \in[\omega]^{\omega}$ such that for each $i \in A, f \neq f_{s(i)}$, and for each $i, j \in A$, if $i<j$, then $\Delta\left(f, f_{s(i)}\right)<\Delta\left(f, f_{s(j)}\right)$ and $n(i)<n(j)$.

Now, since $f_{s(\omega+1)} \neq f_{s(\omega)}$, there must be $k \in\{\omega, \omega+1\}$ such that $f_{s(k)} \neq f$. Fix such a $k \in\{\omega, \omega+1\}$. Let $l=f_{s(k)}\left(\Delta\left(f_{s(k)}, f\right)\right)$. Choose $i \in A$ such that $\Delta\left(f, f_{s(i)}\right)>\Delta\left(f_{s(k)}, f\right)$ and $n(i)>l$. Then $\Delta\left(f_{s(k)}, f_{s(i)}\right)=\Delta\left(f_{s(k)}, f\right)$. However, $f_{s(k)}\left(\Delta\left(f_{s(k)}, f_{s(i)}\right)\right)=l<n(i)$, contradicting $c(\{s(i), s(k)\})=1$. This contradiction finishes the proof.

Observe that properties of $\mathbb{S}$ such as coherence are not used in the proof of Theorem 28 ; we only need that every element of $\mathbb{S}$ has infinitely many immediate successors in $\mathbb{S}$. 
It is also worth pointing out the following corollary of the proof of Theorem 28 as it shows that while in the model of $\operatorname{PFA}(\mathbb{S})$ the partition relation $\omega_{1} \rightarrow\left(\omega_{1}, \omega+2\right)^{2}$ fails, forcing with the coherent Suslin tree $\mathbb{S}$ recuperates it.

Theorem 31. If there is a Suslin tree then $\omega_{1} \nrightarrow\left(\omega_{1}, \omega+2\right)^{2}$.

Although Theorem 14 shows that the statement that $\omega_{1} \rightarrow\left(\omega_{1}, \alpha\right)^{2}$ for all $\alpha<\omega_{1}$ is not equivalent to $\mathfrak{p}>\omega_{1}$ over ZFC+PID, it does not give much further information.

Problem 32. Find a cardinal invariant $\mathfrak{x}$ so that the statement that $\omega_{1} \rightarrow\left(\omega_{1}, \alpha\right)^{2}$ for all $\alpha<\omega_{1}$ is equivalent to $\mathfrak{x}>\omega_{1}$ over PID.

The following result that comes from Theorem 6 and the proof of Theorem 5 above gives a partial answer to this problem.

Theorem 33 (Todorcevic). Assume PID. The following are equivalent:

(1) $\mathfrak{b}>\omega_{1}$.

(2) $\omega_{1} \rightarrow\left(\omega_{1}, \omega+2\right)^{2}$.

This leads us to the following version of Problem 32.

Problem 34. Is the statement that $\omega_{1} \rightarrow\left(\omega_{1}, \alpha\right)^{2}$ for all $\alpha<\omega_{1}$ equivalent to $\mathfrak{b}>\omega_{1}$ over PID?

Note that if, under PID, the cardinal invariant inequality $\mathfrak{b}>\omega_{1}$ does not correspond to $\omega_{1} \rightarrow\left(\omega_{1}, \alpha\right)^{2}$, there must be a minimal $\alpha<\omega_{1}$ with this property. The proof of Theorem 5 actually shows that such $\alpha$ is quite large.

\section{PID and five cofinal types}

Recall that by Theorem 4 above, under PID $+\mathfrak{p}>\omega_{1}, 1, \omega, \omega_{1}, \omega \times \omega_{1}$, and $\left[\omega_{1}\right]^{<\omega}$ are the only cofinal types of directed sets of size at most $\aleph_{1}$. In this section, we find a cardinal invariant which captures, in the sense described in the introduction, this statement. This cardinal invariant is not one of the naturally occurring ones. Rather it is the minimum of two cardinal invariants, the well-known bounding number $\mathfrak{b}$ and another cardinal (see Definition 37 below) which has not been investigated as throughly.

Definition 35. Let $\langle D, \leq\rangle$ be a directed set and suppose $X \subset D$. We say that $X$ is pseudobounded if $\forall A \in[X]^{\omega} \exists B \in[A]^{\omega}[B$ is bounded in $D]$. D is said to be $\sigma$-pseudobounded if $D=\bigcup_{n \in \omega} X_{n}$, where for each $n \in \omega, X_{n}$ is pseudobounded in $D$.

If $\mathcal{I}$ is an ideal on a set $X$, then $\langle\mathcal{I}, \subset\rangle$ is a directed poset. Throughout this section, we view ideals on $\omega$ (or on any countable set) as directed posets ordered by inclusion. Recall that an ideal $\mathcal{I}$ on a countable set $X$ is said to be tall if $\mathcal{I}$ is a proper ideal on $X$ (which means $X \notin \mathcal{I}$ ) and $\forall Y \in[X]^{\omega} \exists Z \in[Y]^{\omega}[Z \in \mathcal{I}]$. As always, an ideal on $X$ is required to contain $[X]^{<\omega}$.

Lemma 36. Let $\mathcal{I}$ be a tall ideal on $\omega$ such that $\langle\mathcal{I}, \subset\rangle$ is $\sigma$-pseudobounded. Then $\left[\omega_{1}\right]^{<\omega} \underline{E}_{T}\langle\mathcal{I}, \subset\rangle$ and $\langle\mathcal{I}, \subset\rangle \mathbb{Z}_{T} \omega \times \omega_{1}$. 
Proof. It is clear that $\left[\omega_{1}\right]^{<\omega} \mathbb{Z}_{T}\langle\mathcal{I}, \subset\rangle$ because $\mathcal{I}$ is $\sigma$-pseudobounded. Suppose for a contradiction that $\langle\mathcal{I}, \subset\rangle \leq_{T} \omega \times \omega_{1}$. Then there exists $\left\{X(n, \alpha): n<\omega \wedge \alpha<\omega_{1}\right\} \subset \mathcal{I}$ such that for any $A \in[\omega]^{\omega}$ and $\left\{X_{n}: n \in A\right\} \subset\left[\omega_{1}\right]^{\omega_{1}},\left\{X(n, \alpha): n \in A \wedge \alpha \in X_{n}\right\}$ is cofinal in $\mathcal{I}$. First of all since $\mathcal{I}$ is proper, for each $n \in \omega$, there must be $k_{n} \in \omega$ and $X_{n} \in\left[\omega_{1}\right]^{\omega_{1}}$ such that $\forall \alpha \in X_{n}\left[k_{n} \notin X(n, \alpha)\right]$. Since $\mathcal{I}$ is tall there is $A \in[\omega]^{\omega}$ such that $\left\{k_{n}: n \in A\right\} \in \mathcal{I}$ (either $\left\{k_{n}: n \in \omega\right\}$ is finite, in which case $A=\omega$ works, or else use tallness). Now $\left\{X(n, \alpha): n \in A \wedge \alpha \in X_{n}\right\}$ is cofinal in $\mathcal{I}$. So there is $n \in A$ and $\alpha \in X_{n}$ such that $\left\{k_{m}: m \in A\right\} \subset X(n, \alpha)$. However, $k_{n} \notin X(n, \alpha)$.

For any directed set $D$, and $X$ a directed cofinal subset of $D, D \equiv_{T} X$. Therefore, if $\mathcal{I}$ is a tall $\sigma$-pseudobounded ideal on $\omega$ and $X \subset \mathcal{I}$ is cofinal, directed, and has size at most $\omega_{1}$, then $\langle X, \subset\rangle$ is not Tukey equivalent to any of $1, \omega, \omega_{1}, \omega \times \omega_{1}$, and $\left[\omega_{1}\right]^{<\omega}$.

Definition 37. $\operatorname{cof}\left(\mathcal{F}_{\sigma}\right)$ is the least $\kappa$ such that there exists a tall, $\sigma$-pseudobounded $F_{\sigma}$ ideal $\mathcal{I}$ on $\omega$ and a directed cofinal $X \subset \mathcal{I}$ such that $|X|=\kappa$.

It is clear that $\omega_{1} \leq \operatorname{cof}\left(\mathcal{F}_{\sigma}\right) \leq \mathfrak{c}$. It is also easy to see that $\operatorname{cov}(\mathcal{M}) \leq \operatorname{cof}\left(\mathcal{F}_{\sigma}\right)$. Later in this section, we will prove that $\mathfrak{b}$ and $\operatorname{cof}\left(\mathcal{F}_{\sigma}\right)$ are independent, even assuming PID. We do not know whether the same cardinal invariant is obtained if the requirement that $\mathcal{I}$ be $\sigma$-pseudobounded is dropped from the definition of $\operatorname{cof}\left(\mathcal{F}_{\sigma}\right)$. This is closely related to the well-known question of whether every tall $F_{\sigma}$ ideal on $\omega$ is either $\sigma$ pseudobounded or Tukey equivalent to $[\mathfrak{c}]^{<\omega}$.

Conjecture 38. Let $\operatorname{cof}^{*}\left(\mathcal{F}_{\sigma}\right)$ be the least $\kappa$ such that there exists a tall $F_{\sigma}$ ideal $\mathcal{I}$ on $\omega$ and a directed cofinal $X \subset \mathcal{I}$ such that $|X|=\kappa$. Then $\operatorname{cof}\left(\mathcal{F}_{\sigma}\right)=\operatorname{cof}^{*}\left(\mathcal{F}_{\sigma}\right)$.

There is another way to think about the cardinals $\operatorname{cof}\left(\mathcal{F}_{\sigma}\right)$ and $\operatorname{cof}^{*}\left(\mathcal{F}_{\sigma}\right)$. For any ideal $\mathcal{I}$ on $\omega$, let $\operatorname{cof}(\mathcal{I})$ denote

$$
\min \{|X|: X \subset \mathcal{I} \wedge X \text { is directed and cofinal in }\langle\mathcal{I}, \subset\rangle\} .
$$

Then $\operatorname{cof}^{*}\left(\mathcal{F}_{\sigma}\right)$ is the minimal value taken by $\operatorname{cof}(\mathcal{I})$ as $\mathcal{I}$ runs over all the tall $F_{\sigma}$ ideals, and $\operatorname{cof}\left(\mathcal{F}_{\sigma}\right)$ is the minimal value as $\mathcal{I}$ runs over all the tall, $\sigma$-pseudobounded $F_{\sigma}$ ideals.

The possible values of $\operatorname{cof}(\mathcal{I})$ for various definable ideals $\mathcal{I}$ on $\omega$ have been investigated by several people. For instance, Louveau and Velickovic [10] proved that if $\mathcal{I}$ is any tall Borel non- $F_{\sigma}$ ideal, then $\operatorname{cof}(\mathcal{I}) \geq \mathfrak{d}$. Other notable results include the observations that $\operatorname{cof}(\mathcal{Z})=\operatorname{cof}(\mathcal{N})$ and $\operatorname{cof}(\mathcal{E D})=\mathfrak{c}$, where $\mathcal{Z}$ is the ideal of subsets of $\omega$ of asymptotic density 0 and $\mathcal{E} \mathcal{D}$ is the ideal on $\omega \times \omega$ generated by the vertical columns and graphs of functions. The cardinal $\operatorname{cof}^{*}\left(\mathcal{F}_{\sigma}\right)$ was investigated in a recent paper by Hrušák et al. [7]. They proved that $\operatorname{cof}^{*}\left(\mathcal{F}_{\sigma}\right)<\mathfrak{b}$ is consistent with ZFC. One part of the proof of Theorem 46 is based on their work. We refer the reader to [7] and the references there for more on the possible values of $\operatorname{cof}(\mathcal{I})$ for definable ideals $\mathcal{I}$. An argument similar to the proof in Case II of Theorem 39 was independently given by Borodulin-Nadzieja and Chodounský in a recent paper [2] to prove that under PID every $\omega_{1}$-tower is Hausdorff iff $\mathfrak{b}>\omega_{1}$.

Theorem 39. Assume PID. The following are equivalent:

(1) $\min \left\{\mathfrak{b}, \operatorname{cof}\left(\mathcal{F}_{\sigma}\right)\right\}>\omega_{1}$.

(2) $1, \omega, \omega_{1}, \omega \times \omega_{1}$, and $\left[\omega_{1}\right]^{<\omega}$ are the only cofinal types of directed sets of size at most $\aleph_{1}$. 
Proof. By the results of Todorčević in [18] it follows that if $\mathfrak{b}=\omega_{1}$, then there is a directed set of size $\omega_{1}$ whose cofinal type is different from any of $1, \omega, \omega_{1}, \omega \times \omega_{1}$, and $\left[\omega_{1}\right]^{<\omega}$.

Next, suppose that $\operatorname{cof}\left(\mathcal{F}_{\sigma}\right)=\omega_{1}$. Let $\mathcal{I}$ and $X \subset \mathcal{I}$ witness this. Then $|X|=\omega_{1}$, and by Lemma 36 , the cofinal type of $\langle X, \subset\rangle$ is not one of $1, \omega, \omega_{1}, \omega \times \omega_{1}$, and $\left[\omega_{1}\right]^{<\omega}$. This proves $\neg(1) \Longrightarrow \neg(2)$.

For the other direction, we assume (1) and prove (2). It is easy to see (for example, see [8]) that if $D$ is a directed set of size at most $\aleph_{1}$ and $D \Varangle_{T} \omega_{1} \times \omega$, then $D \equiv_{T} 1$, or $D \equiv_{T} \omega$, or $D \equiv_{T} \omega_{1}$. Therefore, fixing a directed set $D$ with $|D| \leq \aleph_{1}$, it is sufficient to show that either $D$ contains an uncountable set $X$ all of whose infinite subsets are unbounded (in which case $\left[\omega_{1}\right]^{<\omega} \equiv_{T} D$ ) or else that $D=\bigcup_{n \in \omega} X_{n}$ where for each $n \in \omega$ and each $A \in\left[X_{n}\right]^{\omega}, A$ is bounded in $D$ (in which case $D \leq_{T} \omega_{1} \times \omega$ ). The proof of this proceeds in two cases. We first make some preliminary remarks. For $x \in D, \operatorname{pred}(x)$ denotes $\{y \in D: y \leq x\}$. Let $A \subset D$. Define the trace of $D$ on $A$, $\operatorname{tr}(D, A)=\{B \subset A: \exists x \in D[B \subset \operatorname{pred}(x)]\}$. Note that since $D$ is directed, $\operatorname{tr}(D, A)$ is an ideal on $A$.

Case I: For each $A \in[D]^{\leq \omega}, \operatorname{tr}(D, A)$ is not tall. Let $\mathcal{I}=\left\{A \in[D]^{\leq \omega}: \forall x \in\right.$ $D[|A \cap \operatorname{pred}(x)|<\omega]\}$. It is easy to see that $\mathcal{I}$ is an ideal. To see that it is a P-ideal, fix $\left\{A_{n}: n \in \omega\right\} \subset \mathcal{I}$. Without loss of generality, the $A_{n}$ are pairwise disjoint and infinite. For each $x \in D$ and $n \in \omega$, put $\operatorname{pred}(x, n)=\operatorname{pred}(x) \cap A_{n}$; this is a finite subset of $A_{n}$. As $|D| \leq \omega_{1}<\mathfrak{b}$, it is possible to find finite sets $F_{n} \subset A_{n}$ such that

$$
\forall x \in D \forall^{\infty} n \in \omega\left[\operatorname{pred}(x, n) \subset F_{n}\right] .
$$

Putting $A=\bigcup_{n \in \omega}\left(A_{n} \backslash F_{n}\right)$, it is clear that $A \in \mathcal{I}$ and that $\forall n \in \omega\left[A_{n} \subset^{*} A\right]$. Now, if there is an uncountable $X \subset D$ such that $[X]^{\leq \omega} \subset \mathcal{I}$, then it is clear that every infinite subset of $X$ is unbounded in $D$, and therefore $D \equiv_{T}\left[\omega_{1}\right]^{<\omega}$. So suppose that there exist $\left\{X_{n}: n \in \omega\right\}$ which are pairwise disjoint such that $D=\bigcup_{n \in \omega} X_{n}$, and $\forall n \in \omega\left[\left[X_{n}\right]^{\omega} \cap \mathcal{I}=0\right]$. We claim that for each $n \in \omega$, and each $A \in\left[X_{n}\right]^{\omega}$, $A$ is bounded in $D$. This is sufficient to show that $D \leq_{T} \omega_{1} \times \omega$. Fix $n \in \omega$ and $A \in\left[X_{n}\right]^{\omega}$. The hypothesis of Case I implies that either $\operatorname{tr}(D, A)$ is not a proper ideal on $A$ or that there exists $C \in[A]^{\omega}$ such that $\forall B \in \operatorname{tr}(D, A)[|C \cap B|<\omega]$. Suppose for a moment that $\exists C \in[A]^{\omega} \forall B \in \operatorname{tr}(D, A)[|C \cap B|<\omega]$. Then for any $x \in D, \operatorname{pred}(x) \cap C$ is finite, whence $C \in \mathcal{I}$. However, this means that $C \in \mathcal{I} \cap\left[X_{n}\right]^{\omega}$, contradicting $\mathcal{I} \cap\left[X_{n}\right]^{\omega}=0$. Therefore, it must be the case that $\operatorname{tr}(D, A)$ is not a proper ideal on $A$. So $A \in \operatorname{tr}(D, A)$, whence $A$ is bounded. This completes Case I.

Case II: There exists $A \in[D]^{\omega}$ for which $\mathcal{J}=\operatorname{tr}(D, A)$ is a tall ideal on $A$. In particular, $\mathcal{J}$ is a proper ideal on $A$. Identifying $A$ with a copy of $\omega$, it makes sense to talk about the descriptive complexity of $\mathcal{J}$. Put $X=\{\operatorname{pred}(x) \cap A: x \in D\} \subset \mathcal{J}$, and note that $X$ is a cofinal subset of $\langle\mathcal{J}, \subset\rangle$ of size at most $\omega_{1}$. Moreover, $X$ is directed. To see this if $x, y \in D$, then choosing $z \in D$ such that $x, y \leq z$, it is clear that $\operatorname{pred}(z) \cap A \in X$, and that $\operatorname{pred}(x) \cap A \subset \operatorname{pred}(z) \cap A$ and $\operatorname{pred}(y) \cap A \subset \operatorname{pred}(z) \cap A$. Define $\mathcal{I}=\left\{\mathcal{F} \in[\mathcal{J}]^{\leq \omega}: \forall B \in \mathcal{J}[|\mathcal{F} \cap \mathcal{P}(B)|<\omega]\right\}$. Using the fact that $\mathcal{J}$ has a cofinal subset of size at most $\omega_{1}$ and the hypothesis that $\mathfrak{b}>\omega_{1}$, it is easy to check that $\mathcal{I}$ is a P-ideal. First suppose there is an uncountable $\mathcal{G} \subset \mathcal{J}$ such that $[\mathcal{G}]^{\leq \omega} \subset \mathcal{I}$. For each $B \in \mathcal{G}$ choose $x_{B} \in D$ such that $B \subset \operatorname{pred}\left(x_{B}\right)$. Let $\mathcal{H} \subset \mathcal{G}$ be infinite (not necessarily countable). We claim that $\left\{x_{B}: B \in \mathcal{H}\right\}$ is unbounded in $D$. For if not, 
then fix $x \in D$ such that $x \geq x_{B}$, for all $B \in \mathcal{H}$, and note that $\operatorname{pred}(x) \cap A \in \mathcal{J}$. Now for any $B \in \mathcal{H}, B \subset \operatorname{pred}\left(x_{B}\right) \subset \operatorname{pred}(x)$, and so $B \in \mathcal{P}(\operatorname{pred}(x) \cap A)$. Thus if $\mathcal{L} \in[\mathcal{H}]^{\omega}$, then $\mathcal{L} \cap \mathcal{P}(\operatorname{pred}(x) \cap A)$ is infinite, whence $\mathcal{L} \notin \mathcal{I}$, contradicting $[\mathcal{G}]^{\leq \omega} \subset \mathcal{I}$. It follows that $\left\{x_{B}: B \in \mathcal{G}\right\}$ is an uncountable subset of $D$, and that no infinite subset of it is bounded in $D$.

Next, suppose that there exist $\left\{\mathcal{G}_{n}: n \in \omega\right\}$ which are pairwise disjoint such that $\mathcal{J}=\bigcup_{n \in \omega} \mathcal{G}_{n}$ and $\forall n \in \omega\left[\left[\mathcal{G}_{n}\right]^{\omega} \cap \mathcal{I}=0\right]$. We first claim that each $\mathcal{G}_{n}$ is pseudobounded in $\mathcal{J}$, so that $\mathcal{J}$ is $\sigma$-pseudobounded. Fix $n \in \omega$ and let $\mathcal{F} \in\left[\mathcal{G}_{n}\right]^{\omega}$. Since $\mathcal{F} \notin \mathcal{I}$ and $\mathcal{F} \in[\mathcal{J}]^{\leq \omega}$, there exists $B \in \mathcal{J}$ such that $\mathcal{F} \cap \mathcal{P}(B)$ is infinite. It is clear that $\mathcal{L}=\mathcal{F} \cap \mathcal{P}(B) \in[\mathcal{F}]^{\omega}$ and that for all $C \in \mathcal{L}, C \subset B$, whence $\mathcal{L}$ is bounded in $\mathcal{J}$. It now follows from the assumption that $\operatorname{cof}\left(\mathcal{F}_{\sigma}\right)>\omega_{1}$ that $\mathcal{J}$ is not an $F_{\sigma}$ ideal (with respect to the natural topology on $\mathcal{P}(A)$ ). For each $n \in \omega$, let $\mathcal{H}_{n}$ be the closure of $\mathcal{G}_{n}$ with respect to the usual topology on $\mathcal{P}(A)$. So there is $n \in \omega$ such that $\mathcal{H}_{n} \not \subset \mathcal{J}$. Fix such $n$ and fix $C \in \mathcal{H}_{n} \backslash \mathcal{J}$. Let $\left\{a_{i}: i \in \omega\right\}$ enumerate $A$. Choose $\left\{B_{m}: m \in \omega\right\} \subset \mathcal{G}_{n}$ such that for each $m \in \omega, B_{m} \cap\left\{a_{i}: i<m\right\}=C \cap\left\{a_{i}: i<m\right\}$ and moreover $\forall i<m\left[B_{i} \neq B_{m}\right]$. Thus $\left\{B_{m}: m \in \omega\right\} \in\left[\mathcal{G}_{n}\right]^{\omega}$, and so there exists $B \in \mathcal{J}$ for which $\exists^{\infty} m \in \omega\left[B_{m} \subset B\right]$. We claim that this implies that $C \subset B$. Indeed if $a_{i} \in C$ for some $i \in \omega$, then choose $m>i$ such that $B_{m} \subset B$. Then it is clear that $a_{i} \in B_{m} \subset B$. Thus it follows that $C \in \mathcal{J}$, a contradiction. Since we have a contradiction from the second alternative of PID, it must be that in Case II the first alternative always occurs. This finishes the proof.

A noteworthy feature of this result is that the cardinal $\operatorname{cof}\left(\mathcal{F}_{\sigma}\right)$ speaks about the cofinal structure of definable ideals of size continuum while (2) of Theorem 39 is part of the general theory of cofinal types.

Corollary 40. PFA(S) implies that the coherent Suslin tree $\mathbb{S}$ forces that $1, \omega, \omega_{1}$, $\omega \times \omega_{1}$, and $\left[\omega_{1}\right]^{<\omega}$ are the only cofinal types of directed sets of size at most $\aleph_{1}$.

Proof. It is a theorem of Todorčević $[22]$ that if $\mathbf{V}$ satisfies $\operatorname{PFA}(\mathbb{S})$ and if $G$ is $(\mathbf{V}, \mathbb{S})$ generic, then in $\mathbf{V}[G]$, both PID and $\mathfrak{b}>\omega_{1}$ hold. So in view of Theorem 39 , it suffices to check that in $\mathbf{V}[G], \operatorname{cof}(\mathcal{J})>\omega_{1}$ for any tall $F_{\sigma}$ ideal $\mathcal{J}$. As $\mathbb{S}$ does not add any reals, all $F_{\sigma}$ ideals in $\mathbf{V}[G]$ are coded in $\mathbf{V}$. So fix $\mathcal{J} \in \mathbf{V}$ a code for an $F_{\sigma}$ ideal, and suppose $\Vdash " \mathcal{J}$ is tall." Let $\left\{\stackrel{\circ}{x}_{\alpha}: \alpha<\omega_{1}\right\} \subset \mathbf{V}^{\mathbb{S}}$ with $\Vdash$ " $\stackrel{\circ}{\alpha}_{\alpha} \in \mathcal{J}$," for each $\alpha<\omega_{1}$. We claim that $\Vdash\left\{\stackrel{\circ}{x}_{\alpha}: \alpha<\omega_{1}\right\}$ is not cofinal in $\mathcal{J}$. Fix $p \in \mathbb{S}$. For each $q \leq p$ and $\alpha<\omega_{1}$ find $x(\alpha, q) \in \mathcal{J}$ and $r(\alpha, q) \leq q$ such that $r(\alpha, q) \Vdash \stackrel{\circ}{x}_{\alpha}=x(\alpha, q)$. Since $\mathfrak{p}>\omega_{1}$ holds in $\mathbf{V}$ and since $\mathcal{J}$ is a proper ideal, find $x \in[\omega]^{\omega}$ such that $\forall \alpha<\omega_{1} \forall q \leq p\left[x \subset^{*}(\omega \backslash x(\alpha, q))\right]$. Since $\Vdash$ " $\mathcal{J}$ is tall," there is $y \in \mathcal{J} \cap[x]^{\omega}$. Now it is clear that for each $\alpha<\omega_{1} p \Vdash y \not \subset \stackrel{\circ}{x}_{\alpha}$.

Corollary 41. PID $+\operatorname{add}(\mathcal{M})>\omega_{1}$ implies that $1, \omega, \omega_{1}, \omega \times \omega_{1}$, and $\left[\omega_{1}\right]^{<\omega}$ are the only cofinal types of directed sets of size at most $\aleph_{1}$.

Next, we work toward showing that $\mathfrak{b}$ and $\operatorname{cof}\left(\mathcal{F}_{\sigma}\right)$ are mutually independent even in the presence of PID

Definition 42. A sequence of natural numbers $I=\left\langle k_{n}: n \in \omega\right\rangle$ is called an interval partition if $k_{0}=0$ and $\forall n \in \omega\left[k_{n}<k_{n+1}\right]$. For an interval partition $I$ and $n \in \omega$, let $I_{n}=\left[k_{n}, k_{n+1}\right)$. Let $I$ be an interval partition such that $\forall n \in \omega\left[\left|I_{n}\right| \geq 2^{n}\right]$. In the 
rest of this section, polynomial means a polynomial with integer co-efficients. Define $\mathcal{I}_{\text {poly }}(I)$ to be

$\left\{A \subset \omega\right.$ : there is a polynomial $p(n)$ such that $\left.\forall n \in \omega\left[\left|I_{n} \cap A\right| \leq p(n)\right]\right\}$.

It is clear that this is an $F_{\sigma}$ ideal on $\omega$.

Definition 43. A poset $\mathbb{P}$ is said to have the Laver property if for each sequence of finite sets $\langle H(n): n \in \omega\rangle$ in the ground model $\mathbf{V}$, for each $(\mathbf{V}, \mathbb{P})$-generic $G$, and for each $f \in \mathbf{V}[G] \cap \prod_{n \in \omega} H(n)$, there is a $K \in \mathbf{V} \cap \prod_{n \in \omega} \mathcal{P}(H(n))$ such that $\forall n \in \omega[|K(n)| \leq n+1 \wedge f(n) \in K(n)]$.

The following is a special case of a result of Hrušák et al. [7].

Lemma 44 (Hrušák, Rojas-Rebolledo, and Zapletal). Fix $I \in \mathrm{V}$. Let $\mathbb{P}$ be any poset with the Laver property. Then $\Vdash$ "V $\cap \mathcal{I}_{\text {poly }}(I)$ is a cofinal subset of $\mathcal{I}_{\text {poly }}(I)$."

Proof. In the ground model $\mathbf{V}$, define for each polynomial $p$ and $n \in \omega$, the set $H(p, n)=\left\{s \subset I_{n}:|s| \leq p(n)\right\}$. Let $G$ be $(\mathbf{V}, \mathbb{P})$ generic and let $A \in \mathbf{V}[G] \cap \mathcal{I}_{\text {poly }}(I)$. Let $p$ be a polynomial witnessing this. Define $F_{A} \in \prod_{n \in \omega} H(p, n)$ by $F_{A}(n)=$ $A \cap I_{n}$. By the Laver property, find $K \in \mathbf{V} \cap \prod_{n \in \omega} \mathcal{P}(H(p, n))$ such that $\forall n \in$ $\omega\left[|K(n)| \leq n+1 \wedge F_{A}(n) \in K(n)\right]$. Working in $\mathbf{V}$ define $s_{n}=\bigcup K(n)$, for each $n \in \omega$. Clearly, $s_{n} \subset I_{n}$ and $\left|s_{n}\right| \leq(n+1) p(n)$. Therefore, $B=\bigcup_{n \in \omega} s_{n} \in \mathbf{V} \cap \mathcal{I}_{\text {poly }}(I)$ and $A \subset B$.

Lemma 45. For any $I$ there exist $\left\{X_{n}: n \in \omega\right\}$ such that $\mathcal{I}_{\text {poly }}(I)=\bigcup_{n \in \omega} X_{n}$ and for each $n \in \omega$, every infinite subset of $X_{n}$ has a further infinite subset that is bounded in $\mathcal{I}_{\text {poly }}(I)$. In other words, $\mathcal{I}_{\text {poly }}(I)$ is $\sigma$-pseudobounded.

Proof. Let $\left\{p_{n}: n \in \omega\right\}$ enumerate all polynomials. Define $X_{n}=\left\{A \in \mathcal{I}_{\text {poly }}(I)\right.$ : $\left.\forall m \in \omega\left[\left|I_{m} \cap A\right| \leq p_{n}(m)\right]\right\}$. It is clear that $\mathcal{I}_{\text {poly }}(I)=\bigcup_{n \in \omega} X_{n}$ and that each $X_{n}$ is a closed subset of $\mathcal{P}(\omega)$. Now fix $n$ and an infinite $Y \subset X_{n}$. Let $A \in X_{n}$ be a complete accumulation point of $Y$. For each $m \in \omega$ choose $B_{m} \in Y$ such that $B_{m} \cap\left(\bigcup_{i \leq m} I_{i}\right)=$ $A \cap\left(\bigcup_{i \leq m} I_{i}\right)$ and moreover $\forall i<m\left[B_{m} \neq B_{i}\right]$. Thus $\left\{B_{m}: m \in \omega\right\} \in[Y]^{\omega}$. For each $i \in \omega$, put $s_{i}=\bigcup_{m \in \omega}\left(B_{m} \cap I_{i}\right)$. It is clear that $s_{i}=\left(\bigcup_{m<i}\left(B_{m} \cap I_{i}\right)\right) \cup\left(A \cap I_{i}\right)$. Therefore $\left|s_{i}\right| \leq(i+1) p_{n}(i)$ and $\bigcup_{m \in \omega} B_{m} \in \mathcal{I}_{\text {poly }}(I)$, as needed.

Theorem 46. Assume that there is a supercompact cardinal $\kappa$. Then PID $+\mathfrak{b}>\omega_{1}$ does not imply $\operatorname{cof}\left(\mathcal{F}_{\sigma}\right)>\omega_{1}$. Also there is a model of $\mathrm{PID}+\operatorname{cof}\left(\mathcal{F}_{\sigma}\right)>\omega_{1}$, where $\mathfrak{d}=\omega_{1} \quad$ (a fortiori $\left.\mathfrak{b}=\omega_{1}\right)$.

Proof. By results of $[1,19]$, if $\mathcal{I}$ is any P-ideal, then there is a proper poset not adding reals, call it $\mathbb{P}_{\mathcal{I}}$, that forces $\mathrm{PID}$ with respect to $\mathcal{I}$. Using a Laver diamond in a ground model satisfying $\mathrm{CH}$, do a Countable Support (CS) iteration $\left\langle\mathbb{P}_{\alpha}, \mathbb{Q}_{\alpha}: \alpha \leq \kappa\right\rangle$ as follows. Given $\mathbb{P}_{\alpha}$, if the Laver diamond picks a $\mathbb{P}_{\alpha}$ name for a P-ideal $\mathcal{I}$, then let

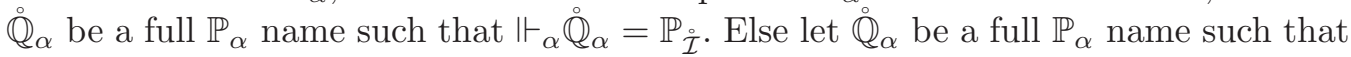
$\Vdash_{\alpha} \stackrel{\mathbb{Q}}{\alpha}_{\alpha}$ is Laver forcing. Note that cofinally often we will have $\Vdash_{\alpha} \stackrel{\mathbb{Q}}{\alpha}_{\alpha}$ is Laver forcing. Also, note that each iterand is forced to have the Laver property, which is preserved in CS iterations. So if $G$ is $\left(\mathbf{V}, \mathbb{P}_{\kappa}\right)$ generic, then in $\mathbf{V}[G], \mathrm{PID}+\mathfrak{b}>\omega_{1}$ holds. Moreover, if $I \in \mathbf{V}$, then by Lemma $44 \mathbf{V} \cap \mathcal{I}_{\text {poly }}(I)$ is cofinal in $\mathbf{V}[G] \cap \mathcal{I}_{\text {poly }}(I)$. By Lemma 45 , 
in $\mathbf{V}[G], \mathcal{I}_{\text {poly }}(I)$ is a tall, $\sigma$-pseudobounded, $F_{\sigma}$ ideal, and $\mathbf{V} \cap \mathcal{I}_{\text {poly }}(I)$ is a directed cofinal subset of $\mathbf{V}[G] \cap \mathcal{I}_{\text {poly }}(I)$ of size $\omega_{1}$. So it witnesses $\operatorname{cof}\left(\mathcal{F}_{\sigma}\right)=\omega_{1}$. This finishes the proof of the first statement.

For the second statement, we use the well-known result of Laflamme [9] that for any ground model $\mathbf{V}$ and any $F_{\sigma}$ ideal $\mathcal{I}$ on $\omega$ belonging to $\mathbf{V}$, there is a proper $\omega^{\omega}$-bounding poset $\mathbb{Q}_{\mathcal{I}} \in \mathbf{V}$ such that $\mathbb{Q}_{\mathcal{I}}$ adds an infinite subset of $\omega$ that is almost disjoint from every member of $\mathbf{V} \cap \mathcal{I}$. Once again, fix a ground model satisfying $\mathrm{CH}$ and a Laver diamond in that ground model. Do a CS iteration $\left\langle\mathbb{P}_{\alpha}, \stackrel{\mathbb{Q}}{\alpha}_{\alpha}: \alpha \leq \kappa\right\rangle$ as

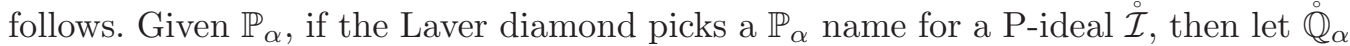
be a full $\mathbb{P}_{\alpha}$ name such that $\vdash_{\alpha} \stackrel{\mathbb{Q}}{\alpha}_{\alpha}=\mathbb{P}_{\mathcal{I}}$. If the Laver diamond picks a pair of $\mathbb{P}_{\alpha}$ names $\langle\stackrel{\circ}{\mathcal{I}}, \stackrel{\circ}{X}\rangle$ such that $\Vdash_{\alpha}$ " $\stackrel{\circ}{\mathcal{I}}$ is a tall $F_{\sigma}$ ideal" and $\Vdash_{\alpha}$ " $\stackrel{\circ}{X}^{\prime} \stackrel{\circ}{\mathcal{I}}$ and $|\stackrel{\circ}{X}|<\kappa$," then let $\mathbb{Q}_{\alpha}$ be a full $\mathbb{P}_{\alpha}$ name for $\mathbb{Q}_{\mathcal{I}}$. If neither of these happens, then $\mathbb{Q}_{\alpha}$ is a full $\mathbb{P}_{\alpha}$ name for the trivial poset. Note that since each iterand is proper and $\omega^{\omega}$-bounding, $\mathbb{P}_{\kappa}$ is $\omega^{\omega}$-bounding. Therefore, if $G$ is $\left(\mathbf{V}, \mathbb{P}_{\kappa}\right)$ generic, then PID $+\mathfrak{d}=\omega_{1}$ holds in $\mathbf{V}[G]$. Also in $\mathbf{V}[G]$, if $\mathcal{I}$ is a tall $F_{\sigma}$ ideal and $X \subset \mathcal{I}$ is of size at most $\omega_{1}$, then there exists $a \in[\omega]^{\omega}$ such that $\forall x \in X[|x \cap a|<\omega]$. This implies that $X$ is not cofinal in $\mathcal{I}$. Therefore, $\operatorname{cof}\left(\mathcal{F}_{\sigma}\right)>\omega_{1}$ holds in $\mathbf{V}[G]$.

\section{Acknowledgements}

The first author was partially supported by a National University of Singapore research grant. The second author was partially supported by NSERC.

\section{References}

[1] U. Abraham and S. Todorčević, Partition properties of $\omega_{1}$ compatible with CH, Fund. Math. 152(2) (1997), 165-181.

[2] P. Borodulin-Nadzieja and D. Chodounský, Hausdorff gaps and towers in $\mathcal{P}(\omega) /$ Fin, preprint.

[3] C. Brech and S. Todorčević, Biorthogonality in Banach spaces under the P-ideal dichotomy, preprint, 2013.

[4] B. Dushnik and E.W. Miller, Partially ordered sets, Amer. J. Math. 63 (1941), 600-610.

[5] P. Erdös and R. Rado, A partition calculus in set theory, Bull. Amer. Math. Soc. 28 (1956), 426-438.

[6] P. Hájek, M.S.V.J. Vanderwerff and V. Zizler, Biorthogonal systems in Banach spaces, CMS Books in Mathematics/Ouvrages de Mathématiques de la SMC, Vol. 26, Springer, New York, 2008.

[7] M. Hrušák, D. Rojas-Rebolledo and J. Zapletal, Cofinalities of Borel ideals, MLQ Math. Log. Q. 60(1-2) (2014), 31-39.

[8] J.R. Isbell, The category of cofinal types. II, Trans. Amer. Math. Soc. 116 (1965), 394-416.

[9] C. Laflamme, Zapping small filters, Proc. Amer. Math. Soc. 114(2) (1992), 535-544.

[10] A. Louveau and B. Velickovic, Analytic ideals and cofinal types, Ann. Pure Appl. Logic 99(1-3) (1999), 171-195.

[11] A. Máté, A partition relation for Souslin trees, Trans. Amer. Math. Soc. 268(1) (1981), $143-149$.

[12] D. Raghavan, P-ideal dichotomy and weak squares, J. Symbolic Logic 78(1) (2013), 157-167.

[13] W. Sierpinski, Sur une problème de la théorie des relations, Ann. Sc. Norm. Super. Pisa, Ser. 2 2 (1933), 239-242.

[14] S. Todorčević, Stationary sets, trees, and continuums, Publ. Inst. Math. (Beograd) (N.S.) 29(43) (1981), 249-262.

[15] S. Todorčević, Forcing positive partition relations, Trans. Amer. Math. Soc. 280(2) (1983), $703-720$.

[16] S. Todorčević, Directed sets and cofinal types, Trans. Amer. Math. Soc. 290(2) (1985), 711-723. 
[17] S. Todorčević, Partition relations for partially ordered sets, Acta Math. 155(1-2) (1985), 1-25.

[18] S. Todorčević, Partition problems in topology, Contemporary Mathematics, Vol. 84, American Mathematical Society, Providence, RI, 1989.

[19] S. Todorčević, A dichotomy for P-ideals of countable sets, Fund. Math. 166(3) (2000), 251-267.

[20] S. Todorčević, Biorthogonal systems and quotient spaces via Baire category methods, Math. Ann. 335(3) (2006), 687-715.

[21] S. Todorčević, Combinatorial dichotomies in set theory, Bull. Symbolic Logic 17(1) (2011), $1-72$.

[22] S. Todorčević, Forcing with a coherent Souslin tree, preprint, 2011.

[23] S. Todorčević and V. Torres Pérez, Conjectures of Rado and Chang and special Aronszajn trees, MLQ Math. Log. Q. 58(4-5) (2012), 342-347.

[24] J.W. Tukey, Convergence and uniformity in topology, Annals of Mathematics Studies, Vol. 2, Princeton University Press, Princeton, NJ, 1940.

[25] M. Viale, A family of covering properties, Math. Res. Lett. 15(2) (2008), 221-238.

Department of Mathematics, National University of Singapore, Singapore 119076 , SINGAPORE

E-mail address: raghavan@math.nus.edu.sg

$U R L:$ http://www.math.toronto.edu/raghavan

Department of Mathematics, University of Toronto, Toronto M5S 2E4, Canada

Institut de Mathématique de Jussieu, UMR 7586, Case 247, 4 Place Jussieu, 75252 Paris Cedex, France

E-mail address: stevo@math.toronto.edu, todorcevic@math.jussieu.fr 
